\title{
Critical Boundary Sine-Gordon Revisited
}

\author{
M. Hasselfield ${ }^{1}$, Taejin Lee ${ }^{1,2,3}$, G.W. Semenoff $f^{1,4}$ and P.C.E. Stamp ${ }^{1}$ \\ ${ }^{1}$ Pacific Institute for Theoretical Physics \\ and \\ Department of Physics and Astronomy, University of British Columbia \\ 6224 Agricultural Road, Vancouver, British Columbia V6T 1Z1, Canada \\ 2 Department of Physics, Kangwon University, Chuncheon 200-701 Korea \\ 3 Asia Pacific Center for Theoretical Physics, Pohang 790-784 Korea \\ ${ }^{4}$ Institute des Hautes Études Scientifiques \\ Le Bois-Marie 35, F-91440 Bures-sur-Yvette, France
}

August 20, 2018

\begin{abstract}
We revisit the exact solution of the two space-time dimensional quantum field theory of a free massless boson with a periodic boundary interaction and self-dual period. We analyze the model by using a mapping to free fermions with a boundary mass term originally suggested in ref. [24. We find that the entire $\operatorname{SL}(2, \mathrm{C})$ family of boundary states of a single boson are boundary sine-Gordon states and we derive a simple explicit expression for the boundary state in fermion variables and as a function of sine-Gordon coupling constants. We use this expression to compute the partition function. We observe that the solution of the model has a strong-weak coupling generalization of T-duality. We then examine a class of recently discovered conformal boundary states for compact bosons with radii which are rational numbers times the self-dual radius. These have simple expression in fermion variables. We postulate sine-Gordon-like field theories with discrete gauge symmetries for which they are the appropriate boundary states.
\end{abstract}




\section{Introduction and Summary}

Boundary conformal field theory consisting of a single boson field is of interest in a wide array of contexts. In condensed matter physics it describes the dissipative quantum mechanics of a particle in a one-dimensional periodic potential [1, 2, 3, 4, and elaborations of it in Josephson junction arrays [5, 6, 7] and the dissipative Hofstadter problem [8, 9]. It also arises in analysis of the Kondo problem [10, 11, the study of one-dimensional conductors [12, tunneling between Hall edge states [13], and junctions of quantum wires [14]. In string theory, boundary conformal field theories are solutions of classical open string field theory and those with boundary operators describe open strings in background fields [15, 16, 17]. In particular, a marginal, periodic boundary interaction which is termed the "rolling tachyon" gives a description of the process of tachyon condensation in string theories with unstable D-branes [18. The relationships between all of these theories are not always trivial, and in this Paper we revisit the boundary Sine-Gordon model from a string theory perspective. We shall see that our results clearly have interesting implications for some of the related models (particularly the condensed matter ones), but to properly elaborate on these is beyond the scope of the present work.

Recently, two of the authors have discussed the rolling tachyon boundary state by fermionizing the rolling tachyon boundary conformal field theory and then finding the boundary states using fermion variables [19]. Here we shall extend that work, which constructed the boundary state for the half-brane, to study the full set of conformal boundary states which can be constructed for a theory with a single free boson in the bulk. These states are parameterized by $\mathrm{SL}(2, \mathrm{C})$ group elements and we find a simple mapping of the $\mathrm{SL}(2, \mathrm{C})$ matrices onto the coupling constants of the boundary sine-Gordon theory. The fermion representation of this problem has some advantages which we exploit, particularly for constructing boundary states and computing partition functions. In addition, the SL(2,C) symmetry and Kac-Moody algebra which appear at the self-dual radius has a simple linear representation in terms of fermion variables. In this language, different boundary conditions are simply related by global $\mathrm{SL}(2, \mathrm{C})$ transformations of the boundary states. In particular, T-duality, which interchanges Neumann and Dirichlet coordinates, is a simple chiral rotation. We shall use this observation to show that a generalization of T-duality persists when the critical boundary sine-Gordon interaction is turned on and appears as a strong coupling-weak coupling duality of the critical theory.

We shall discuss conformal boundary states which have recently been shown to appear for compact bosons with rational radii [20]. These also have a simple description in fermion variables as projections of the $\mathrm{SL}(2, \mathrm{C})$ boundary states onto a sectors of the theory with some fixed fermion charges. These projections can be viewed as Gauss' law constraints for a discrete gauge symmetry. We use our mapping of boundary states onto boundary sine-Gordon models to suggest a gauged boundary sine-Gordon field theory for which these are the appropriate boundary states.

Our central result is an improved understanding of how the three parameters $(g, \bar{g}, A)$ in the boundary sine-Gordon action of eq. (11) below correspond to the three marginal boundary deformations of $c=1$ conformal field theories 21] and how they are encoded 
in boundary states when the boundary states are written in fermion variables. We also find that the fermion representation gives a solution of the theory which has a different dependence on the sine-Gordon coupling constants than the one which was found in the bosonic approach and which is used extensively in the literature on tachyon condensation. (See for example the scalar amplitude in eq.(10) below compared with appropriate Wick rotation with eq. (4.10) in ref. [18.) The dependence of the solution of a field theory on its bare couplings is generally not universal, it is a matter of the detailed definition of the theory, including cutoff and renormalization procedures. However, knowledge of how the solution of a theory depends on the bare coupling constants can be important in condensed matter applications where the physical input to the construction of a microelectronic device, for example, are the bare parameters. Our solution presents one such definition which is an alternative to the other known ones, and which we believe is natural.

\subsection{Critical Boundary Sine-Gordon Theory}

Consider the theory of a single boson field on a strip with time coordinate $t \in(-\infty, \infty)$, space $s \in(0, \pi)$ and with action

$$
S=\frac{1}{4 \pi} \int_{-\infty}^{\infty} d t \int_{0}^{\pi} d s\left(\partial_{t} X^{2}-\partial_{s} X^{2}\right)-\int_{-\infty}^{\infty} d t\left(\frac{g}{2} e^{i X(t, 0)}+\frac{\bar{g}}{2} e^{-i X(t, 0)}+A \partial_{t} X(t, 0)\right)
$$

This is a free field theory in the bulk of the strip, with equation of motion

$$
\left(\partial_{t}^{2}-\partial_{s}^{2}\right) X(t, s)=0
$$

and is an interacting field theory by virtue of the nonlinear boundary condition at $s=0$,

$$
-\partial_{s} X(t, 0)+i \frac{g}{2} e^{i X(t, 0)}-i \frac{\bar{g}}{2} e^{-i X(t, 0)}=0
$$

At the other boundary, $s=\pi$, we will consider an array of possible boundary conditions: the Dirichlet condition, $\partial_{t} X(t, \pi)=0$, the Neumann condition $\partial_{s} X(t, \pi)=0$ and the addition of a periodic boundary operator leading to a condition of type (2), perhaps with different parameters.

We will allow $g$ and $\bar{g}$ to be independent complex numbers, not necessarily complex conjugates of each other. Of course, for the Hamiltonian to be Hermitian would require the special case $\bar{g}=g^{*}$. The boundary interaction potential is known to be an exactly marginal operator and (11) is a conformal field theory for all values of $g$ and $\bar{g}$.

This boundary conformal field theory is tuned to lie at the critical point which separates two distinct behaviors of a one-dimensional boson with a periodic boundary potential. In one regime, the boundary potential would be a relevant operator. Its effect would be to localize the boundary value of $X$ near the minima of the potential. There, the coupling constant in the boundary interaction would have a non-zero beta function. The renormalization group flow would be toward the conformal invariant Dirichlet boundary condition which fixes the boundary $X$ at a particular location. 
In the other regime, the boundary potential would be an irrelevant operator. The boundary value of $X$ would be mobile and would fluctuate amongst the minima of the periodic potential. In that case, the renormalization group flow of the coupling constant would be toward the translation invariant and conformal invariant Neumann boundary condition where complete translation invariance would be restored.

In the boundary conformal field theory (11), which sits between these two phases, the boundary potential is an exactly marginal operator. This exactly solvable theory describes a family of critical states which, as we vary the constants $g, \bar{g}$, interpolate between the two kinds of behavior. $X$ becomes delocalized when $g \sim 0 \sim \bar{g}$, where the boundary condition reverts to Neumann. It is completely localized when the coupling constant is increased to $\pi|g|=1=\pi|\bar{g}|$ where the boundary state becomes Dirichlet and indeed pins the boundary value of $X$ at the minima of the potential. When $\pi|g|>1, \pi|\bar{g}|>1$ the theory is apparently non-unitary.

The last, topological term in the action (11) does not influence the equations of motion or the boundary condition. ${ }^{1}$ It does not destroy the conformal symmetry of the model but we shall see that the spectrum generally depends on it. (It was shown in refs. [22, 23] that the conformal dimensions of operators can depend on such a term.) For a noncompact boson, it can be interpreted as a Bloch wave-number. To see this, first consider the case with $A=0$ and the boson $X(t, s)$ taking values on the entire real line. The potential is periodic. We assume that the boundary condition at $s=\pi$ is also periodic. Then the translation $X(t, s) \rightarrow X(t, s)+2 \pi$ is a discrete symmetry. The eigenstates of the Hamiltonian must carry a representation of the symmetry group. Since the group is Abelian, irreducible representations are one-dimensional. Therefore a wave-functional eigenstate of the Hamiltonian must transform as

$$
\Psi_{E}[X+2 \pi]=e^{-2 \pi i A} \Psi_{E}[X]
$$

where $A$ takes values between zero and one. We can fix $A$, by considering all states which transform by the same phase, $e^{-2 \pi i A}$. This is the content of Bloch's theorem and the phase is called the Bloch wave-number. In this sector of the theory, we can pass to strictly periodic wave-functionals by a canonical transformation, which acts on the wavefunctional by a unitary operator. The operator is formed by exponentiating the generator of the transformation,

$$
\Psi_{E}[X]=e^{-i A X(0,0)} \tilde{\Psi}_{E}[X] \quad, \quad \tilde{\Psi}_{E}[X+2 \pi]=\tilde{\Psi}_{E}[X]
$$

Under such a canonical transformation, the Lagrangian is replaced by itself plus the total time derivative of the generating function,

$$
S=\int d t L \rightarrow \int d t\left\{L-\frac{d}{d t}[A X(t, 0)]\right\}
$$

This restores the topological parameter $A$ in the action. In solid-sate physics the functions $\Psi_{E}[X]$ are known as Wannier functions. We see that the parameter $A$ coincides with the

\footnotetext{
${ }^{1}$ The other possible simple boundary term which one might think of adding is $\int d t \partial_{s} X(t, 0)$. This term could be eliminated by using the boundary condition (2), and re-defining the coupling constants $g$ and $\bar{g}$.
} 
Bloch wave-number of the boson field on its target space. Once it appears explicitly in the action, when we compute the partition function or the correlators of periodic operators, the boson field can be treated as if it were compact, that is, as if it were subject to the periodic identification $X(t, s) \sim X(t, s)+2 \pi$.

If the boson were indeed compact, no physical observable, including matrix elements between different wave-functions can be changed by replacing $X$ by $X+2 \pi$. In this case, all wave-functions must have the behavior (3) with the same parameter $A$. In this case, we would simply leave $A$ as a freely adjustable parameter of the theory.

For a non-compact boson, we would compute the partition function or the correlator of periodic operators with $A$ fixed as if we were computing the same object for a compact boson. Then we would integrate the partition function or correlator over the allowed values of $A$, from zero to one.

The periodicity of the potential is also compatible with a boson which is identified with a period larger than the basic one, say $X \sim X+2 \pi N$. To obtain this case, rather than integrating over $A$, we would write $A=n / N+a$ and sum over $n=0,1, \ldots, N-1$. The theory would then be parameterized by another angle with $a \in[0,1 / N)$. In this way one would develop a 'multiple band' theory for this periodic potential- this in principle is quite complicated because of transitions between the bands.

In string theory $A$ is interpreted as a constant gauge potential on the target space, this is the origin of the terminology "Wilson line".

\subsection{Boundary versus bulk operators}

We will apply the boundary state technique to computation of the partition function. We will also find the boundary state in terms of fermion variables. A rough idea of the problem at hand can be gotten by considering the scaling dimensions of the boundary operator. Consider a free scalar field $X$ with Neumann boundary conditions. When $: e^{i X}$ : is an operator living in the bulk of a two-dimensional space it has conformal dimensions $(1 / 4,1 / 4)$, whereas, when it is an operator on the boundary it has dimensions $(1 / 2,1 / 2)$. The difference arises from the Neumann boundary condition which fuses the boundary operator to its image, thereby doubling its dimension.

We shall want to construct boundary states which are coherent states of the bulk operators which satisfy the boundary condition. We shall therefore need the boundary potential to be a fermion bilinear in the bulk as well as on the boundary. A fermion bilinear operator like $\psi_{L}^{\dagger} \psi_{R}$ has dimension $(1 / 2,1 / 2)$ both in the bulk and on the boundary. The boundary dimension $(1 / 2,1 / 2)$ of $: e^{i X}$ : is just right to be a fermion bilinear on the boundary, but its $(1 / 4,1 / 4)$ in the bulk is wrong for a fermion bilinear, it cannot be one in the bulk.

The trick for getting around this difficulty was originally applied to this model by Polchinski and Thorlacius [24] who introduced a second free boson $Y$ with Dirichlet boundary conditions. Then $: e^{i Y}$ : is a bulk operator of dimension $(1 / 4,1 / 4)$ and a boundary operator of dimension $(0,0)$. The composite $: e^{i(X+Y)}:$ has precisely the property that we want, it has dimension $(1 / 2,1 / 2)$ both in the bulk and on the boundary and can therefore be a fermion bilinear in both environments. Because of the Dirichlet boundary condition, 
$Y=0$ on the boundary, therefore, up to a multiplicative constant, $: e^{i(X+Y)}:$ coincides with the operator of the boundary potential, $: e^{i X}:$, at the boundary.

Because $Y$ is a free boson with a simple boundary condition, its dynamics are exactly solvable and its contribution can easily be identified and discarded when computing the partition function or any correlators involving $X$ alone. Using this trick will allow us to fermionize the boundary interaction and to construct boundary states. Most of the analysis of the present paper centers on applying this idea to the boundary sine-Gordon theory.

\subsection{Summary of partition functions}

\subsubsection{Annulus amplitude}

One of our main results is the general formula for the partition function. Consider the situation where there is a boundary sine-Gordon interaction similar to the one in eqn.(1) on each of the two boundaries with parameters $\left(g_{i}, \bar{g}_{i}, A_{i}\right)$ and $i=1,2$. In the path integral representation of the partition function, the time is Euclidean and is periodic, so the spacetime has the topology of an annulus.

If $H$ is the Hamiltonian of the field theory in (1), we shall show that the partition function, defined as $Z_{B_{1} B_{2}}[\beta]=\operatorname{Tr} e^{-\beta H}$ is given by the expression

$$
Z_{B_{1} B_{2}}[\beta]=\int_{0}^{1} d A e^{\beta / 24} \sum_{n \in \mathcal{Z}} e^{-\beta(n+\delta)^{2}} \prod_{k=1}^{\infty} \frac{1}{1-e^{-\beta k}}
$$

where

$$
\delta[A, g, \bar{g}]=\frac{1}{2 \pi} \cos ^{-1}\left\{\cos (2 \pi A) \sqrt{1-\pi^{2} g_{1} \bar{g}_{1}} \sqrt{1-\pi^{2} g_{2} \bar{g}_{2}}+\frac{\pi^{2}}{2}\left(g_{1} \bar{g}_{2}+\bar{g}_{1} g_{2}\right)\right\}
$$

Here $A=A_{1}-A_{2}$.

The result in (6) is presented for a non-compact boson. If the boson were compact, with the self-dual radius, we would omit the integral over $A$, which would then remain as a parameter of the theory. In that case, by letting the parameters $\left(g_{i}, \bar{g}_{i}, A_{i}\right)$ be complex numbers, and by varying them we sweep over the full set of $\operatorname{SL}(2, \mathrm{C})$ conformal boundary states, and therefore the full set of boundary conformal field theories with the periodicity $X \sim X+2 \pi$.

From (6) and (7) we see that the ground state energy is

$$
E_{0}=\delta^{2}-\frac{1}{24}
$$

where we choose the branch of delta with smallest absolute value. This energy is generally real when $\bar{g}_{i}=g_{i}^{*}$ and when $A$ is real. For a compact boson it depends on the Wilson line parameter $A$ in a simple way. If the boson is non-compact, we should instead think of (8) as an energy band, which varies over the energies in the band as we vary $A$ over its range. For an example, see Fig. 1. 
The energies of excited states are

$$
E=(n+\delta)^{2}+N-\frac{1}{24}
$$

where $n$ is any integer and $N$ is a non-negative integer. The degeneracy of each state is given by $p[N]$, the number of partitions of $N$ (where we define $p[0]=1$ ).

Other partition functions are easily obtained as limits of (6) . The boundary condition reduces to a Neumann condition if we put $g=0=\bar{g}$ and a Dirichlet condition if we put $g=\bar{g}^{*}$ and $|g|=1 / \pi$. In the second case, the partition function no longer depends on $A$ and the phase of $-g$ gives the D-brane position on the circle, or if the theory is non-compact, the positions of an infinite periodic array of D-branes.

\subsubsection{Disc amplitude}

For some applications, for example the dissipative particle, path integral representation of the partition function has the field theory living on a Euclidean space with the geometry a disc 4]. The boundary interaction lies on the boundary of the disc. The most accessible quantity in the boundary state formalism is the expectation value of a vertex operator such as $e^{i k_{L} X_{L}+i k_{R} X_{R}}$ which is inserted into the bulk of the disc. Expectation values of these operators are computed explicitly in Section 5 for all values of $\left(k_{L}, k_{R}\right)$ and have a very simple expression. With them, we can find the inner product of the bulk boson position eigenstate $\mid X_{0 L}, X_{0 R}>$ and the boundary state

$$
|B>=| X_{0 L}, X_{0 R}><X_{0 L}, X_{0 R} \mid B>+(\text { oscillators }) \mid X_{0 L}, X_{0 R}>
$$

For a non-compact boson, the result is

$$
2^{\frac{1}{4}}<X_{0}=X_{0 L}+X_{0 R} \mid B>=\frac{1}{2 \pi}\left[\frac{1}{1+\pi g e^{i X_{0}}}+\frac{1}{1+\pi \bar{g} e^{-i X_{0}}}-1\right]
$$

For a compact boson, with the identification $X \sim X+2 \pi$, the result is similar, but also retains a contribution of the wrapped states,

$$
\begin{aligned}
& 2^{\frac{1}{4}}<X_{0 L}, X_{0 R} \mid B>=\frac{1}{2 \pi}\left[\frac{1}{1+\pi g e^{i X_{0}}}+\frac{1}{1+\pi \bar{g} e^{-i X_{0}}}+\frac{1}{1-\sqrt{1-\pi^{2} g \bar{g}} e^{i \hat{X}_{0}+2 \pi i A}}\right. \\
& \left.+\frac{1}{1-\sqrt{1-\pi^{2} g \bar{g}} e^{-i \hat{X}_{0}-2 \pi i A}}-3\right]
\end{aligned}
$$

where $X_{0}=X_{0 L}+X_{0 R}$ is position on the circle and $\hat{X}_{0}=X_{0 L}-X_{0 R}$ is position on the dual circle. Note that $\hat{X}_{0}$ is redundant with the Wilson line $A$. In Section 4, we will discuss why this is expected. 
For boundary states where the boson is compact with a rational radius, $X \sim X+$ $2 \pi M / N$

$$
\begin{aligned}
2^{\frac{1}{4}} & <X_{0 L}, X_{0 R} \mid B>=\frac{1}{2 \pi}\left[\frac{1}{1+(\pi g)^{M} e^{i M X_{0}}}+\frac{1}{1+(\pi \bar{g})^{M} e^{-i M X_{0}}}+\right. \\
& \left.+\frac{1}{1-\left(1-\pi^{2} g \bar{g}\right)^{N / 2} e^{i N \hat{X}_{0} 2 \pi i N A}}+\frac{1}{1-\left(1-\pi^{2} g \bar{g}\right)^{N / 2} e^{-i N \hat{X}_{0}-2 \pi i N A}}-3\right]
\end{aligned}
$$

Here, the exponents $M$ and $N$ arise from the fact that these boundary states are very similar to the one which gives (11), the main difference being that states with unwanted momentum and wrapping numbers have been eliminated. This cull of states leads to a similar cull of powers of $e^{i X_{0}}$ and $e^{i \hat{X}_{0}}$ leaving only multiples of $M$ and $N$ respectively.

\subsubsection{Duality}

Eqs. (11) and (12) have an interesting apparent symmetry. If we make the interchange of coordinate and dual coordinate,

$$
X_{0} \leftrightarrow \hat{X}_{0}
$$

that is normally made to implement T-duality, and we also make the replacements

$$
\begin{aligned}
\pi g & \leftrightarrow-\sqrt{1-\pi^{2} g \bar{g}} e^{2 \pi i A} \\
\pi \bar{g} & \leftrightarrow-\sqrt{1-\pi^{2} g \bar{g}} e^{-2 \pi i A}
\end{aligned}
$$

we see that the amplitudes in eqs. (11) and (12) retain the same form. In fact, the entire energy spectrum (9) is invariant. This replacement - which at the operator level is $\left(X_{L}, X_{R}\right) \rightarrow\left(X_{L},-X_{R}\right)$ - is a symmetry of the field theory which can easily be seen in the boundary state formalism. It is a generalization of T-duality and it maps to each other the weak coupling and strong coupling regimes of the conformal field theory.

\section{Boundary states}

We shall apply the boundary state technique to the problem of computing the partition function,

$$
Z[\beta]=\operatorname{Tr} e^{-\beta H}
$$

where $H$ is the Hamiltonian of the field theory in (11). When this partition function is computed using the path integral formulation, the action has periodically identified Euclidean time $\tau \in(0, \beta)$ so that the space-time geometry is a Euclidean cylinder.

The boundary state technique interchanges the space and time coordinates on the cylinder to compute the same partition function as a matrix element of the Euclidean time

evolution operator for a free boson on a cylinder between boundary states which encode all of the information about the boundary conditions,

$$
Z[\beta]=<B_{1}\left|e^{-\frac{2 \pi^{2}}{\beta} \hat{H}}\right| B_{2}>
$$


Now, the space variable is periodic, $\sigma \sim \sigma+\beta$ and the time variable goes between the boundaries of the cylinder $0 \leq \tau \leq \pi$. In the following we shall use conformal invariance to re-scale the dimensions to match usual conventions for open and closed strings where $0 \leq \sigma<2 \pi$. We have anticipated this re-scaling in the Euclidean time parameter, $\alpha=\frac{2 \pi^{2}}{\beta}$, in (14). The Hamiltonian $\hat{H}$ in (14) is that of the free boson on a Euclidean cylinder, which has action

$$
\hat{S}=\frac{1}{4 \pi} \int_{-\infty}^{\infty} d \tau \int_{0}^{2 \pi} d \sigma\left(\partial_{\tau} X^{2}+\partial_{\sigma} X^{2}\right)
$$

with $X(\tau, 2 \pi)=X(\tau, 0)$. The conformal transformation $z=e^{\tau+i \sigma}, \bar{z}=e^{\tau-i \sigma}$ maps the cylinder to the complex plane with boundaries of the cylinder mapped to the origin and the circle at infinity. Radial quantization of (15) has the equation of motion

$$
\frac{\partial}{\partial \bar{z}} \frac{\partial}{\partial z} X(z, \bar{z})=0
$$

which is solved by $X(z, \bar{z})=X_{L}(z)+X_{R}(\bar{z})$ with the mode expansion

$X_{L}(z)=\frac{1}{\sqrt{2}}\left(x_{L}-i p_{L} \ln z+i \sum_{n \neq 0} \frac{\alpha_{n}}{n} z^{-n}\right), X_{R}(\bar{z})=\frac{1}{\sqrt{2}}\left(x_{R}-i p_{R} \ln \bar{z}+i \sum_{n \neq 0} \frac{\tilde{\alpha}_{n}}{n} \bar{z}^{-n}\right)$

where the non-vanishing commutators $\left[x_{L}, p_{L}\right]=i,\left[x_{R}, p_{R}\right]=i,\left[\alpha_{m}, \alpha_{n}\right]=m \delta_{m+n}$, $\left[\tilde{\alpha}_{m}, \tilde{\alpha}_{n}\right]=m \delta_{m+n}$ represent the canonical commutator in Euclidean time,

$$
\left[X(0, \sigma), \partial_{\tau} X\left(0, \sigma^{\prime}\right)\right]=2 \pi \delta\left(\sigma-\sigma^{\prime}\right)
$$

The Hamiltonian is

$$
\hat{H}=\frac{1}{2} p_{L}^{2}+\frac{1}{2} p_{R}^{2}+\sum_{n=1}^{\infty}\left(\alpha_{-n} \alpha_{n}+\tilde{\alpha}_{-n} \tilde{\alpha}_{n}\right)-\frac{1}{12}
$$

The vacuum $\mid p_{L}, p_{R}>$ is an eigenstate of $p_{L}$ and $p_{R}$ and is annihilated by all positively moded oscillators $\alpha_{n}$ and $\tilde{\alpha}_{n}$ with $n>0$. For a compact boson with the same period as the potential, the momenta are quantized,

$$
X \sim X+2 \pi \rightarrow \frac{1}{\sqrt{2}}\left(p_{L}+p_{R}\right)=\text { integer } \quad, \frac{1}{\sqrt{2}}\left(p_{L}-p_{R}\right)=\text { integer }
$$

The boundary states are squeezed states which are annihilated by the operator which would be the boundary condition for the field theory on the strip (with $\sigma$ and $\tau$ interchanged). Examples are the Neumann $\mid N>$ and Dirichlet $\mid D>$ states which obey the Neumann and Dirichlet boundary conditions ${ }^{2}$

$$
\left(\partial_{\tau} X_{L}(0, \sigma)+\partial_{\tau} X_{R}(0, \sigma)\right)\left|N>=0 \quad\left(\partial_{\tau} X_{L}(0, \sigma)-\partial_{\tau} X_{R}(0, \sigma)\right)\right| D>=0
$$

\footnotetext{
${ }^{2}$ In the following, we shall sometimes use the Neuman and Dirichlet boundary conditions in the stronger forms

$$
\left(X_{L}(0, \sigma)-X_{R}(0, \sigma)\right)\left|N>=0, \quad\left(X_{L}(0, \sigma)+X_{R}(0, \sigma)\right)\right| D>=0
$$

These conditions are solved by the boundary states $\mid N>$ and $\mid D>$ in (21a) and (21b) with $A=0$.
} 
respectively. These are solved by ${ }^{3}$

$$
\begin{aligned}
& \left|N>=2^{-\frac{1}{4}} \prod_{n>0} e^{-\frac{1}{n} \alpha_{-n} \tilde{\alpha}_{-n}} \sum_{p_{L}} e^{-2 \pi i A \sqrt{2} p_{L}}\right| p_{L},-p_{L}> \\
& \left|D>=2^{-\frac{1}{4}} \prod_{n>0} e^{\frac{1}{n} \alpha_{-n} \tilde{\alpha}_{-n}} \sum_{p_{L}} e^{-2 \pi i A \sqrt{2} p_{L}}\right| p_{L}, p_{L}>.
\end{aligned}
$$

These states are related to each other by T-duality, $\left(X_{L}, X_{R}\right) \rightarrow\left(X_{L},-X_{R}\right)$, which interchanges Neuman and Dirichelt boundary conditions. $A$ is a parameter of the solutions. It is the Wilson line for the Neuman state, analogous to the parameter in (11). For the Dirichlet state, it is the position of the D-brane, $X(0, \sigma)|D>=-2 \pi(A \bmod n)| D>$.

The boundary state which obeys

$$
\left(-\partial_{\tau} X(0, \sigma)+i \frac{g}{2} e^{i X(0, \sigma)}-i \frac{\bar{g}}{2} e^{-i X(0, \sigma)}\right) \mid B>=0
$$

is also known explicitly. It has been constructed in ref. 25] using the level-1 SU(2) KacMoody algebra which appears at the self-dual radius (18). ${ }^{4}$ More recently, a large class of correlation functions has been computed [26. Almost simultaneously with the appearance of ref. [25, the model (11) (with $\bar{g}=g^{*}$ ) was also solved in ref. [24] by mapping it onto a theory of free fermions with a boundary mass operator. They did not construct boundary states but did compute the partition function and boundary S-matrix and their results agreed with those of ref. [25]. A limit of this model where $\bar{g}=0$ was solved by construction of the boundary state in fermion variables in ref. 19. In the following, we will employ the techniques of ref. [19] to find the boundary state $|B\rangle$ in terms of fermion variables and compute the partition function $Z[\beta]$ as a function of $g, \bar{g}$ and $A$ for various combinations of boundary conditions.

\subsection{Fermionization at the self-dual radius}

Let us re-write the problem of finding the boundary state $\mid B>$ in terms of fermion variables. Following refs. [24] and [19], the first step is to double the degrees of freedom by introducing another boson field $Y$ which obeys a Dirichlet boundary condition on all boundaries. The Euclidean action becomes

$$
S=\frac{1}{4 \pi} \int_{-\infty}^{\infty} d \tau \int_{0}^{2 \pi} d \sigma\left(\partial_{\tau} X^{2}+\partial_{\sigma} X^{2}+\partial_{\tau} Y^{2}+\partial_{\sigma} Y^{2}\right)
$$

and the boundary condition is now the two equations

$$
\begin{aligned}
{\left[-\frac{1}{2 \pi} \partial_{\tau} X(0, \sigma)+i \frac{g}{2} e^{i X(0, \sigma)}-i \frac{\bar{g}}{2} e^{-i X(0, \sigma)}\right]|B, D\rangle } & =0 \\
Y(0, \sigma)|B, D\rangle & =0 .
\end{aligned}
$$

\footnotetext{
${ }^{3}$ The awkward factor of $2^{-\frac{1}{4}}$ in the normalization of these states arises from the need to produce the correct partition function for the boson theory on the strip.

${ }^{4}$ The same current algebra is also the vertex operator algebra of the discrete states of special 'primary operators of non-compact bosons [27] which, if we do not compactify the boson, still play a special role. See ref. 25] for some discussion of this point.
} 
The theory for $Y$ is decoupled and is exactly solvable. Its contribution to quantities such as the partition function is easily identified, factored out and discarded to return to the theory with $X$ alone. The presence of $Y$ is needed to make the mapping to fermion variables described below. It allows us to form the variables

$$
\phi_{1}=\frac{1}{\sqrt{2}}(X+Y) \quad, \quad \phi_{2}=\frac{1}{\sqrt{2}}(X-Y)
$$

with which the mapping to fermions is defined:

$$
\begin{aligned}
& \psi_{1 L}(z)=\zeta_{1 L}: e^{-\sqrt{2} i \phi_{1 L}(z)}: \quad, \quad \psi_{1 L}^{\dagger}(z)=: e^{\sqrt{2} i \phi_{1 L}(z)}: \zeta_{1 L}^{\dagger} \\
& \psi_{2 L}(z)=\zeta_{2 L}: e^{\sqrt{2} i \phi_{2 L}(z)}: \quad, \quad \psi_{2 L}^{\dagger}(z)=: e^{-\sqrt{2} i \phi_{2 L}(z)}: \zeta_{2 L}^{\dagger} \\
& \psi_{1 R}(\bar{z})=\zeta_{1 R}: e^{\sqrt{2} i \phi_{1 R}(\bar{z})}: \quad, \quad \psi_{1 R}^{\dagger}(\bar{z})=: e^{-\sqrt{2} i \phi_{1 R}(\bar{z})}: \zeta_{1 R}^{\dagger} \\
& \psi_{2 R}(\bar{z})=\zeta_{2 R}: e^{-\sqrt{2} i \phi_{2 R}(\bar{z})}: \quad, \quad \psi_{2 R}^{\dagger}(\bar{z})=: e^{\sqrt{2} i \phi_{2 R}(\bar{z})}: \zeta_{2 R}^{\dagger}
\end{aligned}
$$

where $\zeta_{a L / R}$ are co-cycles. Co-cycles must be introduced in order to make the fermion fields anti-commute with each other. An explicit representation of the co-cycles constructed from the zero modes of the bosons, $\sqrt{2} \phi_{a L / R}=\varphi_{a L / R}-i \pi_{a L / R} \ln z+\ldots$ is given in ref. [19],

$\zeta_{1 L}=\zeta_{1 R}=\exp \left(-i \frac{\pi}{2}\left(\pi_{1 L}+\pi_{1 R}+2 \pi_{2 L}+2 \pi_{2 R}\right)\right), \zeta_{2 L}=\zeta_{2 R}=\exp \left(-i \frac{\pi}{2}\left(\pi_{2 L}+\pi_{2 R}\right)\right)$

The fermion bilinear operators are

$$
\begin{array}{rll}
: \psi_{1 L}^{\dagger}(z) \psi_{1 L}(z):=i \sqrt{2} \partial_{\tau} \phi_{1 L}(z) & , \quad: \psi_{2 L}^{\dagger}(z) \psi_{2 L}(z):=-i \sqrt{2} \partial_{\tau} \phi_{2 L}(z) \\
: \psi_{1 R}^{\dagger}(\bar{z}) \psi_{1 R}(\bar{z}):=-i \sqrt{2} \partial_{\tau} \phi_{1 R}(\bar{z}) & , \quad: \psi_{2 R}^{\dagger}(\bar{z}) \psi_{2 R}(\bar{z}):=i \sqrt{2} \partial_{\tau} \phi_{2 R}(\bar{z}) .
\end{array}
$$

The fermion action on the infinite cylinder is

$$
S=\frac{i}{2 \pi} \int_{-\infty}^{\infty} d \tau \int_{0}^{2 \pi} d \sigma\left(\psi_{a L}^{\dagger}\left(\partial_{\tau}+i \partial_{\sigma}\right) \psi_{a L}+\psi_{a R}^{\dagger}\left(\partial_{\tau}-i \partial_{\sigma}\right) \psi_{a R}\right)
$$

The fermion field theory has an explicit $S U(2)_{L} \times S U(2)_{R}$ chiral symmetry which, because the currents are holomorphic, extends to a linear representation of two copies of the level-1 $\mathrm{SU}(2)$ Kac-Moody algebra generated by

$$
\begin{array}{r}
J_{L}^{a}(z)=\frac{1}{2}: \psi_{L}^{\dagger}(z) \sigma^{a} \psi_{L}(z): \quad \sigma^{a}=\text { Pauli matrices } \\
J_{L}^{\prime+}(z)=\psi_{L 1}^{\dagger}(z) \psi_{L 2}^{\dagger}(z) \quad, \quad J_{L}^{\prime-}(z)=\psi_{L 2}(z) \psi_{L 1}(z) \quad, \quad J_{L}^{\prime 3}(z)=\frac{1}{2}: \psi_{L}^{\dagger}(z) \psi_{L}(z):
\end{array}
$$

The current algebras generated by $J_{L}^{a}(z)$ and ${J^{\prime}}_{L}^{a}(z)$ are identical to the well-known bosonic current algebras for the $X$ - and $Y$-bosons, respectively. There are also anti-holomorphic currents, $J_{R}^{a}(\bar{z})$ and ${J^{\prime}}_{R}^{a}(\bar{z})$.

The mapping of fermion states to boson states is not generally $1-1$. When the fermions are quantized, the eigenvalues of the fermion number operators, which are identified with 
boson momenta, are spaced by integers. This suggests that the fermions correspond to compact bosons. Indeed, it was shown in ref. [19] that, to obtain the states of the bosons compactified at the self-dual radius (18) and when $Y$ is also identified in the same way, $Y \sim Y+2 \pi$, we must consider fermions in two sectors, the Ramond (R) sector where they all have periodic boundary conditions on the cylinder and the Neveu-Schwarz (NS) sector where all are anti-periodic. Then we must project onto states with even total fermion number. The latter is the analog of the GSO projection of the worldsheet fermions in the Neveu-Schwarz-Ramond formulation of the superstring which obtains the type 0A and $0 \mathrm{~B}$ theories. Indeed, it is known to be the unique projection which produces a modular invariant partition function in $\mathrm{D}=1[28]$.

If the bosons are not compact, we shall first treat them as compact and solve for their partition function which will turn out to depend on the Wilson line variable $A$. Then, in accord with our discussion in Sect. 1.1, we integrate the partition function over allowed values of $A$.

\subsection{Boundary states in fermion variables}

We shall write the boundary conditions in eqs. (24a) and (24b) in fermion variables. The first term is $\partial_{\tau} X(0, \sigma)=\frac{1}{2 i}\left[: \psi_{L}^{\dagger}(0, \sigma) \sigma^{3} \psi_{L}(0, \sigma):-: \psi_{R}^{\dagger}(0, \sigma) \sigma^{3} \psi_{R}(0, \sigma):\right]$. Moreover, we can also use the Dirichlet condition for $Y(24 \mathrm{~b})$ in the boundary interaction,

$$
\begin{aligned}
e^{i X(0, \sigma)}|B, D\rangle & =e^{i(X(0, \sigma)+Y(0, \sigma))}|B, D\rangle \\
& =e^{\sqrt{2} i \phi_{1 L}(0, \sigma)} e^{\sqrt{2} i \phi_{1 R}(0, \sigma)}|B, D\rangle \\
& =Z^{2}: e^{\sqrt{2} i \phi_{1 L}(0, \sigma)}:: e^{\sqrt{2} i \phi_{1 R}(0, \sigma)}:|B, D\rangle \\
& =Z^{2} \psi_{1 L}^{\dagger}(0, \sigma) \zeta_{1 L} \zeta_{1 R}^{\dagger} \psi_{1 R}(0, \sigma)|B, D\rangle \\
& =\frac{1}{2} Z^{2} \psi_{L}^{\dagger}(0, \sigma)\left(1+\sigma^{3}\right) \psi_{R}(0, \sigma) \cdot|B, D\rangle
\end{aligned}
$$

Here, when we use the explicit representation of the co-cycles (27), they cancel. $Z$ is an infinite constant which accounts for the difference between the operators and normal ordered operators. We shall absorb $Z^{2}$ by re-defining the coupling constant $g$. This provides the correct power of the cutoff to make $g$ a marginal coupling and is the only renormalization that is required in this model.

In addition, the last term in the boundary interaction is ${ }^{5}$

$$
e^{-i X(0, \sigma)}|B, D\rangle=e^{-i(X(0, \sigma)-Y(0, \sigma))}|B, D\rangle=\frac{1}{2} Z^{2} \psi_{L}^{\dagger}(0, \sigma)\left(1-\sigma^{3}\right) \psi_{R}(0, \sigma)|B, D\rangle
$$

The boundary state condition (24a) becomes

$$
\left[: \psi_{L}^{\dagger} \sigma^{3} \psi_{L}:-: \psi_{R}^{\dagger} \sigma^{3} \psi_{R}:+\pi g \psi_{L}^{\dagger}\left(1+\sigma^{3}\right) \psi_{R}-\pi \bar{g} \psi_{L}^{\dagger}\left(1-\sigma^{3}\right) \psi_{R}\right]|B, D\rangle=0
$$

${ }^{5}$ Note that we could have also fermionized $e^{-i(X+Y)}$ to get the operator $\psi_{R}^{\dagger} \frac{1}{2}\left(1+\sigma^{3}\right) \psi_{L}$. Consistency requires that this operator, when acting on the boundary state, is equivalent to the one in (32). It is possible to use the solution for $\mid B D>$ that we shall find to demonstrate that this is indeed the case. 
Our task is now to find a state in the fermion theory which solves (33) and retains the Dirichlet boundary condition for the $Y$-boson. It is known that some boundary states are related to each other by global $\mathrm{SU}(2)$ rotations of the left-handed fields. For example, the DD and ND boundary conditions

$$
\begin{aligned}
\left(\psi_{R}(0, \sigma)-\psi_{L}(0, \sigma)\right) \mid D D>=0 \quad & \left(\psi_{R}^{\dagger}(0, \sigma)+\psi_{L}^{\dagger}(0, \sigma)\right) \mid D D>=0 \\
\left(\psi_{R}(0, \sigma)+i \sigma^{1} \psi_{L}(0, \sigma)\right) \mid N D>=0 \quad & \left(\psi_{R}^{\dagger}(0, \sigma)+\psi_{L}^{\dagger}(0, \sigma) i \sigma^{1}\right) \mid N D>=0
\end{aligned}
$$

can be found directly from the boson-fermion mapping in eqs. (26a)-(26d), the $\mathrm{N}$ and $\mathrm{D}$ conditions for $\mathrm{X}$ and $\mathrm{Y}$ in (19) and the co-cycles (27). Solving the simple equations (34a) and (34b) (see ref. 19]) obtains the boundary states in fermion variables, These are identical to the states which would be obtained by the appropriate direct product of the $\mathrm{N}$ and D boundary states which we have written in boson variables in eqs. (21a $-(21 \mathrm{~b})$.

The DD and ND boundary conditions in (34a) and (34b) are related by a global $S U(2)$ rotation. With the appropriate choice of phases, the boundary states must also be related by the same transformation,

$$
\left|D D>=e^{-i \pi J_{L}^{1}}\right| N D>
$$

where $J_{L}^{a}=\oint \frac{d \sigma}{2 \pi} J_{L}^{a}(0, \sigma)$ are the generators of the $S U(2)$ algebra. It was argued in ref. 25] that $\mid B>$ can also be obtained from $\mid N>$ by a global rotation. To examine this possibility in the present context, we begin with the ansatz

$$
\left|B D>=e^{-i \theta \cdot J_{L}}\right| N D>
$$

We shall find that for the most general case, we shall require complex values of $\theta^{a}$, and hence a transformation in $S L(2, C)$. Since $J_{L}^{a}$ operates on the $X$-boson only, the transformation of the $\mid N D>$ state in (36) does not upset the Dirichlet boundary condition for the $Y$-boson. The transformation operates on the fermions by

$$
e^{-i \theta \cdot J_{L}} \psi_{L} e^{i \theta \cdot J_{L}}=U \psi_{L}, e^{-i \theta \cdot J_{L}} \psi_{L}^{\dagger} e^{i \theta \cdot J_{L}}=\psi_{L}^{\dagger} U^{-1}, U=e^{i \theta \cdot \sigma / 2}
$$

The fact that the transformation operates on the $X$-boson only and leaves the $Y$-boson unchanged is encoded in $\operatorname{det}[U]=1$. The postulate (36) is equivalent to the statement that, like DD and ND boundary conditions which are presented in eqs. (34a) and (34b) as the usual gluing conditions of boundary conformal field theory, the boundary state $\mid B D>$ also obeys a gluing condition,

$$
\left(\psi_{R}(0, \sigma)+i \sigma^{1} U \psi_{L}(0, \sigma)\right)\left|B D>=0 \quad, \quad\left(\psi_{R}^{\dagger}(0, \sigma)+\psi_{L}^{\dagger}(0, \sigma) U^{-1} i \sigma^{1}\right)\right| B D>=0
$$

This class of boundary conditions automatically imply that $\mid B D>$ is a conformal boundary state, since the energy momentum tensor is an $S U(2) \times S U(2)$ invariant quadratic in fermion operators,

$$
\left(L_{n}-\tilde{L}_{-n}\right) \mid B D>=0
$$


It is easy to see that the boundary condition (38) solves eq. (33) when $U$ is

$$
U=\left[\begin{array}{cc}
e^{-2 \pi i A} \sqrt{1-\pi^{2} g \bar{g}} & -i \pi g \\
-i \pi \bar{g} & e^{2 \pi i A} \sqrt{1-\pi^{2} g \bar{g}}
\end{array}\right]
$$

The constant $A$ is not determined by eq. (33). However, certain partition functions constructed using the boundary state depend on it. We will argue later that it is indeed the topological parameter of the action (11) and we have anticipated this by the notation. $U \in \mathrm{SL}(2, \mathrm{C})$ is a complex matrix with unit determinant. It is unitary (and the angles in (37) real - this is needed for unitarity of the conformal field theory) when $A$ is real and when $\bar{g}=g^{*}$ and $|g|<1 / \pi$. When the coupling exceeds a critical strength, $|g|>1 / \pi$, the matrix is not unitary.

Note, by comparing with (35), that the state $\mid B D>$ becomes the state $\mid D D>$ when $U=i \sigma^{1}$. By inspecting (39) we see that this happens at the critical coupling strength, when $g=-1 / \pi=\bar{g}$. This can be generalized to $g=-e^{i \phi} / \pi$ and $\bar{g}=-e^{-i \phi} / \pi$. Then, the phase $\phi$ corresponds to the D-brane position on the circle, or if the boson is not compact, the positions of an infinite array of equally spaced D-branes.

We can now construct the boundary state explicitly using the fermions. The quantization of fermions uses the mode expansion

$$
\begin{aligned}
& \psi_{L a}(z)=\sum_{r} \psi_{a, r} z^{-r} \quad, \quad \psi_{R a}(\bar{z})=\sum_{r} \tilde{\psi}_{a, r} \bar{z}^{-r} \\
& \psi_{L a}^{\dagger}(z)=\sum_{r} \psi_{a, r}^{\dagger} z^{-r} \quad, \quad \psi_{R a}^{\dagger}(\bar{z})=\sum_{r} \tilde{\psi}_{a, r}^{\dagger} \bar{z}^{-r}
\end{aligned}
$$

where $r$ are half-odd integers in the NS sector and integers in the R sector. Non-vanishing anti-commutators are

$$
\left\{\psi_{a, r}, \psi_{b, s}^{\dagger}\right\}=\delta_{a b} \delta_{r s}, \quad\left\{\tilde{\psi}_{a, r}, \tilde{\psi}_{b, s}^{\dagger}\right\}=\delta_{a b} \delta_{r s}
$$

The vacuum states are annihilated by all positively moded operators and care in handling the zero modes in the $\mathrm{R}$ sector is required. ${ }^{6}$ In the N-S sector the $\mid B D>$ state is ${ }^{7}$

$$
\left|B D>_{N S}=2^{-\frac{1}{2}} \prod_{r=\frac{1}{2}}^{\infty} \exp \left[\psi_{-r}^{\dagger} U^{-1} i \sigma^{1} \tilde{\psi}_{-r}-\tilde{\psi}_{-r}^{\dagger} i \sigma^{1} U \psi_{-r}\right]\right| 0>
$$

and in the R-sector it is

$$
\left|B D>_{R}=2^{-\frac{1}{2}} \prod_{n=1}^{\infty} \exp \left[\psi_{-n}^{\dagger} U^{-1} i \sigma^{1} \tilde{\psi}_{-n}-\tilde{\psi}_{-n}^{\dagger} i \sigma^{1} U \psi_{-n}\right] \exp \left[\psi_{0}^{\dagger} U^{-1} i \sigma^{1} \tilde{\psi}_{0}\right]\right|-+-+>
$$

${ }^{6}$ We define the $\mathrm{R}$ sector state $\mid---->$ as the one which is annihilated by all positively moded oscillators and the zero mode operators $\left(\psi_{1,0}, \tilde{\psi}_{1,0}, \psi_{2,0}, \tilde{\psi}_{2,0}\right)$, respectively. Then $\psi_{a, 0}^{\dagger}$ or $\tilde{\psi}_{a, 0}^{\dagger}$ operating on this state flips a - to a + in the appropriate position. The phase is defined as having a plus sign when the creation operators are ordered as $\left(\psi_{1,0}^{\dagger}, \tilde{\psi}_{1,0}^{\dagger}, \psi_{2,0}^{\dagger}, \tilde{\psi}_{2,0}^{\dagger}\right)$, In total there are 16 degenerate ground states of the fermion system. For each species of fermion, the fermion number of $\mid \pm>$ is $\pm \frac{1}{2}$.

${ }^{7}$ Some of the phase conventions differ from ref. [19]. 
The factor of $2^{-\frac{1}{2}}$ has the same origin as the factor of $2^{-\frac{1}{4}}$ in the boson boundary states (21a) $-(21 \mathrm{~b})$. The Hamiltonian in the NS and R sectors are

$$
\begin{aligned}
\hat{H}_{N S} & =\sum_{r=\frac{1}{2}}^{\infty} r\left(\psi_{-r}^{\dagger} \psi_{r}+\psi_{-r} \psi_{r}^{\dagger}+\tilde{\psi}_{-r}^{\dagger} \tilde{\psi}_{r}+\tilde{\psi}_{-r} \tilde{\psi}_{r}^{\dagger}\right)-\frac{1}{6} \\
\hat{H}_{R} & =\sum_{n=1}^{\infty} n\left(\psi_{-n}^{\dagger} \psi_{n}+\psi_{-n} \psi_{n}^{\dagger}+\tilde{\psi}_{-n}^{\dagger} \tilde{\psi}_{n}+\tilde{\psi}_{-n} \tilde{\psi}_{n}^{\dagger}\right)+\frac{1}{3}
\end{aligned}
$$

respectively. In the NS sector,

$$
e^{-\alpha \hat{H}}\left|B D>=2^{-\frac{1}{2}} e^{\frac{\alpha}{6}} \prod_{r=\frac{1}{2}}^{\infty} \exp \left[e^{-2 \alpha r} \psi_{-r}^{\dagger} U^{-1} i \sigma^{1} \tilde{\psi}_{-r}-e^{-2 \alpha r} \tilde{\psi}_{-r}^{\dagger} i \sigma^{1} U \psi_{-r}\right]\right| 0>
$$

As a concrete example, we will first compute the matrix element of this state with the $\mid D D>$ boundary state. The $\mid D D>$ state is gotten from $\mid B D>\operatorname{simply}$ by replacing $U$ by $i \sigma^{1}$, to get

$$
N S<D D\left|=2^{-\frac{1}{2}}<0\right| \prod_{r=\frac{1}{2}}^{\infty} \exp \left[-\tilde{\psi}_{r}^{\dagger} \psi_{r}+\psi_{r}^{\dagger} \tilde{\psi}_{r}\right]
$$

(To take the conjugate, we have to know that the conjugate of $\psi_{-r}$ is $\psi_{r}^{\dagger}$, etc.) The partition function of interest is

$$
\begin{aligned}
Z & =<D D\left|e^{-\alpha \hat{H}}\right| B D> \\
& =\frac{1}{2} e^{\frac{\alpha}{6}}<0\left|\prod_{r=\frac{1}{2}}^{\infty} e^{-\psi_{r}^{\dagger} \tilde{\psi}_{r}+\psi_{r}^{\dagger} \tilde{\psi}_{r}} e^{e^{-2 \alpha r} \psi_{-r}^{\dagger} U^{-1} i \sigma^{1} \tilde{\psi}_{-r}-e^{-2 \alpha r} \tilde{\psi}_{-r}^{\dagger} i \sigma^{1} U \psi_{-r}}\right| 0>
\end{aligned}
$$

It is easy to find this matrix element by computing it for one value of $r$ at a time. To simplify further, in Section 3, we shall show that it depends only on the eigenvalues of $-i \sigma^{1} U$ which, since the determinant is one, are a complex number $\zeta$ and its inverse $1 / \zeta$ where

$$
\zeta=\frac{\pi(g+\bar{g})}{2}+i \sqrt{1-\left(\frac{\pi(g+\bar{g})}{2}\right)^{2}}
$$

Note that these eigenvalues do not depend on the parameter $A$ in (39). The NS sector 
partition function is ${ }^{8}$

$$
\begin{aligned}
<D D\left|e^{-\alpha \hat{H}}\right| B D>\left.\right|_{N S} & =\frac{1}{2} e^{\frac{\alpha}{6}} \prod_{r=\frac{1}{2}}^{\infty}\left(1+\zeta e^{-2 \alpha r}\right)^{2}\left(1+\zeta^{-1} e^{-2 \alpha r}\right)^{2} \\
& =\frac{1}{2} e^{\frac{\alpha}{6}}\left[\sum_{n} \zeta^{n} e^{-\alpha n^{2}} \prod_{k=1}^{\infty} \frac{1}{\left(1-e^{-2 \alpha k}\right)}\right]^{2}
\end{aligned}
$$

Similarly, in the R sector, the partition function is

$$
\begin{aligned}
<D D\left|e^{-\alpha \hat{H}}\right| B D>\left.\right|_{R} & =\frac{1}{2} e^{-\frac{\alpha}{3}} \prod_{n=1}^{\infty}\left(1+\zeta e^{-2 \alpha n}\right)^{2}\left(1+\zeta^{-1} e^{-2 \alpha n}\right)^{2} \cdot(1+\zeta)\left(1+\zeta^{-1}\right) \\
& =\frac{1}{2} e^{\frac{\alpha}{6}}\left[\sum_{n} \zeta^{\left(n+\frac{1}{2}\right)} e^{-\alpha\left(n+\frac{1}{2}\right)^{2}} \prod_{k=1}^{\infty} \frac{1}{\left(1-e^{-2 \alpha k}\right)}\right]^{2}
\end{aligned}
$$

In the first line above, the product on the right-hand-side is the contribution of non-zero modes and the last factors are the contribution of the zero modes.

We must still remember to project onto the states whose total fermion number is even. The boundary state $\mid N D>$ has even fermion number and neither the operation of the Hamiltonian nor the $\mathrm{SL}(2, \mathrm{C})$ transformation changes that. Therefore, no projection is needed. The full partition function is obtained by combining the NS and R sectors,

$$
\begin{aligned}
Z & =<D D\left|e^{-\alpha \hat{H}}\right| B D>\left.\right|_{N S}+<D D\left|e^{-\alpha H}\right| B D>\left.\right|_{R} \\
& =\frac{1}{2} e^{\frac{\alpha}{6}}\left(\prod_{k=1}^{\infty} \frac{1}{\left(1-e^{-2 \alpha k}\right)}\right)^{2}\left(\left[\sum_{n} \zeta^{n} e^{-\alpha n^{2}}\right]^{2}+\left[\sum_{n} \zeta^{\left(n+\frac{1}{2}\right)} e^{-\alpha\left(n+\frac{1}{2}\right)^{2}}\right]^{2}\right) \\
& =\frac{1}{2} e^{\frac{\alpha}{6}}\left(\prod_{k=1}^{\infty} \frac{1}{\left(1-e^{-2 \alpha k}\right)}\right)^{2} \sum_{m, n} \zeta^{m-n}\left(e^{-\alpha\left(m^{2}+n^{2}\right)}+e^{-\alpha\left(m+\frac{1}{2}\right)^{2}-\alpha\left(n+\frac{1}{2}\right)^{2}}\right) \\
& =\frac{1}{2} e^{\frac{\alpha}{6}}\left(\prod_{k=1}^{\infty} \frac{1}{\left(1-e^{-2 \alpha k}\right)}\right)^{2} \sum_{m, n} e^{-\frac{1}{2} \alpha(m-n)^{2}} \zeta^{m-n}\left(e^{-\frac{1}{2} \alpha(m+n)^{2}}+e^{-\frac{1}{2} \alpha(m+n+1)^{2}}\right)
\end{aligned}
$$

When $m$ and $n$ run over all of the integers, $m+n, m-n$ run over all pairs of integers where both are even or both are odd and $m+n+1, m-n$ run over all pairs of integers where one is even and the other is odd. Thus, the last expression is

$$
Z=\left(\frac{1}{\sqrt{2}} e^{\frac{\alpha}{12}} \sum_{n} e^{-\frac{1}{2} \alpha n^{2}} \prod_{k=1}^{\infty} \frac{1}{\left(1-e^{-2 \alpha k}\right)}\right) \cdot\left(\frac{1}{\sqrt{2}}{ }^{\frac{\alpha}{12}} \sum_{n} \zeta^{n} e^{-\frac{1}{2} \alpha n^{2}} \prod_{k=1}^{\infty} \frac{1}{\left(1-e^{-2 \alpha k}\right)}\right)
$$

\footnotetext{
${ }^{8}$ We use the Jacobi triple product formula

$$
\sum_{n \in \mathcal{Z}} z^{n} q^{n^{2}}=\prod_{n=0}^{\infty}\left(1-q^{2 n+2}\right)\left(1+z q^{2 n+1}\right)\left(1+z^{-1} q^{2 n+1}\right)
$$
}


The first factor on the right-hand-side is the partition function of the $Y$-boson which we discard. The remaining partition function is

$$
<D\left|e^{-\alpha \hat{H}}\right| B>=\frac{1}{\sqrt{2}} e^{\frac{\alpha}{12}} \sum_{n} \zeta^{n} e^{-\frac{1}{2} \alpha n^{2}} \prod_{k=1}^{\infty} \frac{1}{\left(1-e^{-2 \alpha k}\right)}
$$

Now, we can recover the partition function of the field theory on a strip if, according to (14), we put $\alpha=2 \pi^{2} / \beta$. Then, a modular transformation, Poisson resummation ${ }^{9}$ and using (48) give

$$
Z_{D B}[\beta]=e^{\frac{\beta}{24}} \sum_{n} e^{-\beta(n+\delta)^{2}} \prod_{k=1}^{\infty} \frac{1}{\left(1-e^{-\beta k}\right)} \quad, \quad \delta=\frac{1}{2 \pi} \cos ^{-1} \frac{\pi}{2}(g+\bar{g})
$$

This coincides with the results quoted in [25] and [24] if we make a certain re-definition of the coupling constants $(g, \bar{g})$. We shall discuss this re-definition in the next Section.

\section{Partition functions and spectrum}

In this section, we shall summarize some comments about what we have done so far.

1. Wilson line: The source of the parameter $A$ in (39) is clear. The boundary condition (22) does not fix the dependence of $\mid B>$ on the combination of zero modes $x_{L}-x_{R}$. It is easy to see that, if $\theta^{a}$ can be chosen so that $\left|B D>=e^{-i \theta \cdot J_{L}}\right| N D>$ satisfies the boundary state equation, then

$$
\left|B D>_{a}=e^{-i a J_{L}^{3}} e^{-i \theta \cdot J_{L}} e^{-i a J_{L}^{3}}\right| N D>
$$

also satisfies the same equation. This replaces $2 \pi A$ by $2 \pi A+a$ in the solution (39). To see that this amounts to a translation of $x_{L}-x_{R}$, we simply observe that we can use the Neumann boundary condition to write this state as

$$
\left|B D>_{a}=e^{-i a J_{L}^{3}} e^{-i \theta \cdot J_{L}} e^{-i a J_{R}^{3}}\right| N D>=e^{-i a\left(J_{L}^{3}+J_{R}^{3}\right)} e^{-i \theta \cdot J_{L}} \mid N D>
$$

${ }^{9}$ The Dedekind eta-function which is defined by

$$
\eta(\tau) \equiv e^{2 \pi i \tau / 24} \prod_{n=1}^{\infty}\left(1-e^{2 \pi i \tau n}\right)
$$

has the modular transformations

$$
\eta(\tau+1)=e^{2 \pi i / 24} \eta(\tau), \quad \eta(-1 / \tau)=(-i \tau)^{\frac{1}{2}} \eta(\tau)
$$

The Poisson re-summation is

$$
\sum_{p \in \mathcal{Z}} e^{-a p^{2}+i b p}=\sqrt{\frac{\pi}{a}} \sum_{n \in \mathcal{Z}} e^{-\frac{\pi^{2}}{a}(n+b / 2 \pi)^{2}} .
$$


and that $J_{L}^{3}+J_{R}^{3}$ generates a translation of $x_{L}-x_{R}$, leaving $x_{L}+x_{R}, y_{L}$ and $y_{R}$ and all other boson modes unchanged. If we write $\left|B D>_{A}=e^{-2 \pi i A\left(J_{L}^{3}+J_{R}^{3}\right)}\right| B D>_{0}$, we see that, indeed

$$
e^{-2 \pi i A\left(J_{L}^{3}+J_{R}^{3}\right)}=e^{A \int d \sigma\left(\partial_{\tau} X_{L}-\partial_{\tau} X_{R}\right)}=e^{-i A \oint d \sigma \partial_{\sigma}\left(X_{L}+X_{R}\right)}
$$

inserts the operator $e^{-i A \oint d \sigma \partial_{\sigma}\left(X_{L}+X_{R}\right)}$ at the boundary and produces the topological term in the action (11).

2. Partition function with general boundary states: In ref. [29, 20] it has been argued that the general conformal boundary state of a system which satisfies the consistency conditions of boundary conformal field theory with a single free boson compactified at the self-dual radius is the one obtained by the $\operatorname{SL}(2, \mathrm{C})$ transformation $\left|\theta>=e^{-i \theta \cdot J_{L}}\right| N>{ }^{10}$ It is generally specified by three complex parameters, $\theta^{a}$. When $\theta^{a}$ are complex, the conjugate of this state is defined as $<\theta|\equiv<N| e^{i \theta \cdot J_{L}}$. The partition function between two such states is

$$
<\theta_{1}\left|e^{-\frac{2 \pi^{2}}{\beta} \hat{H}}\right| \theta_{2}>=e^{\frac{\beta}{24}} \sum_{n \in \mathcal{Z}} e^{-\beta(n+\delta)^{2}} \prod_{k=1}^{\infty} \frac{1}{\left(1-e^{-\beta k}\right)}
$$

where $\left(e^{2 \pi i \delta}, e^{-2 \pi i \delta}\right)$ are eigenvalues of $U_{2} U_{1}^{-1}$ and $U_{i}=e^{i \theta_{i} \cdot \sigma / 2}$. The result is quoted in eqs. (6) and (77).

Some interesting special cases can be found by taking limits of eqs. (6) and (77).

- The case where one of the boundaries has a Dirichlet condition, $U_{1}=i \sigma^{1}$, agrees with (54).

- When one of the boundaries has a Neumann boundary condition, $U_{1}=1$, $Z_{N \theta}[\beta]=A_{1}<N\left|e^{-\frac{2 \pi^{2}}{\beta} \hat{H}}\right| B>_{A_{2}}$, is given by (158) with

$$
\delta=\frac{1}{2 \pi} \cos ^{-1}\left(\sqrt{1-\pi^{2} g \bar{g}} \cos 2 \pi\left(A_{1}-A_{2}\right)\right)
$$

- For the half-brane, $g \neq 0$ and $\bar{g}=0$, the partition function is given by eq. (6) with $\delta=\left(A_{1}-A_{2}\right)$. This agrees with the known result that the open-string partition function for the half-brane is independent of $g$. See ref. [32, 33, 34, for a discussion of this point and a computation using the bosonic version of the boundary state.

3. Partition function depends only on eigenvalues: It is easy to see why the partition function (58) should only depend on the eigenvalues of the matrix $U_{2} U_{1}^{-1}$. The boundary state $\mid N D>$ is strictly invariant under the simultaneous left- and right$\operatorname{SL}(2, \mathrm{C})$ rotation $\left|N D>=e^{i \hat{\theta} \cdot J_{R}} e^{-i \theta \cdot J_{L}}\right| N D>$ where $\left(\hat{\theta}_{1}, \hat{\theta}_{2}, \hat{\theta}_{3}\right)=\left(\theta_{1},-\theta_{2},-\theta_{3}\right)$ and

\footnotetext{
${ }^{10}$ For a review of boundary conformal field theory in similar contexts, see [30] and one which discusses the particular boundary states of interest here, see [31.
} 
$\theta^{a}$ are real numbers. The reason for this is the fact that, for example in the NS sector, using fermi statistics, the boundary state can be written as

$$
\left|N D>=\prod_{r=\frac{1}{2}}^{\infty} \exp \left(\psi_{-r}^{\dagger} i \sigma^{1} \tilde{\psi}_{-r}-\tilde{\psi}_{-r}^{\dagger} i \sigma^{1} \psi_{-r}\right)\right| 0>
$$

It is easy to see that, for example $\left[J_{L}^{2}-J_{R}^{2}, \psi_{-r}^{\dagger} i \sigma^{1} \tilde{\psi}_{-r}-\tilde{\psi}_{-r}^{\dagger} i \sigma^{1} \psi_{-r}\right]=0$ Another way to see this is to note that the $N D$ boundary condition for the fermions (34b), which is easily seen to be enforced when operating on the state in (60), is preserved by the simultaneous rotations

$$
\psi_{r} \rightarrow e^{i \theta \cdot \sigma / 2} \psi_{r} \quad, \quad \psi_{r}^{\dagger} \rightarrow \psi_{r}^{\dagger} e^{-i \theta \cdot \sigma / 2} \quad, \quad \tilde{\psi}_{-r} \rightarrow e^{i \hat{\theta} \cdot \sigma / 2} \tilde{\psi}_{-r} \quad \tilde{\psi}_{-r}^{\dagger} \rightarrow \tilde{\psi}_{-r}^{\dagger} e^{-i \hat{\theta} \cdot \sigma / 2}
$$

since $\sigma^{1} e^{i \theta \cdot \sigma / 2} \sigma^{1}=e^{i \hat{\theta} \cdot \sigma / 2}$.

Then,

$$
<N D|\ldots| N D>=<N D\left|e^{-i \hat{\theta} \cdot J_{R}} e^{i \theta \cdot J_{L}} \ldots e^{i \hat{\theta} \cdot J_{R}} e^{-i \theta \cdot J_{L}}\right| N D>
$$

Since $J_{R}^{a}$ commutes with $H$ and $J_{L}^{a}$, the contents of "..." in the above equation, we see that

$$
<N D|\ldots| N D>=<N D\left|e^{i \theta \cdot J_{L}} \ldots e^{-i \theta \cdot J_{L}}\right| N D>
$$

This symmetry implies that the partition function depends only on equivalence classes of pairs of states, with the equivalence relation $U_{2} U_{1}^{-1} \sim g U_{2} U_{1}^{-1} g^{-1}$. Such a conjugation can always be chosen so as to bring the matrix to upper triangular form with diagonal elements the eigenvalues. The equivalence classes are therefore parameterized by the eigenvalues which, since the determinant of $U_{2} U_{1}^{-1}$ must be one, are of the form $(\zeta, 1 / \zeta)$ where $\zeta$ is a non-zero complex number.

4. Relation to previous work: The formal expression for the boundary state is the exponential of the boundary potential acting on the Neumann state as

$$
\left|B>=e^{-\int V(\phi)}\right| N>
$$

In the fermion variables, it is straightforward to show by direct substitution that

$$
\left|B D>=e^{-\int\left(\frac{g}{2} \psi_{L}^{\dagger} \frac{1}{2}\left(1+\sigma^{3}\right) \psi_{R}+\frac{\bar{g}}{2} \psi_{L}^{\dagger} \frac{1}{2}\left(1-\sigma^{3}\right) \psi_{R}\right)}\right| N D>
$$

is a solution of the boundary state equation (33). If we could then use the Neumann boundary condition directly in the operator in the exponent to convert $\psi_{R}$ to $\psi_{L}$ there. The terms in the exponent it would become generators of the global SL $(2, \mathrm{C})$ symmetry and it would then be manifestly clear that $\mid B D>$ is an $\mathrm{SL}(2, \mathrm{C})$ rotation of $\mid N D>$. However, in order to do this, a difficulty with operator ordering must be solved. The authors of ref. 25] give an argument which shows that all of the effects of 
operator ordering can be absorbed into the renormalization of the coupling constant. What this implies is that there exist re-defined coupling constants $g^{\prime}(g, \bar{g}), \bar{g}^{\prime}(g, \bar{g})$ such that

$$
e^{-\int\left(\frac{g}{2} \psi_{L}^{\dagger} \frac{1}{2}\left(1+\sigma^{3}\right) \psi_{R}+\frac{\bar{g}}{2} \psi_{L}^{\dagger} \frac{1}{2}\left(1-\sigma^{3}\right) \psi_{R}\right)}\left|N D>=e^{\chi\left(g^{\prime}, \bar{g}^{\prime}\right)} e^{i \int\left(\frac{g^{\prime}}{2} \psi_{L}^{\dagger} \sigma^{+} \psi_{L}+\frac{\bar{g}}{2} \psi_{L}^{\dagger} \sigma^{-} \psi_{L}\right)}\right| N D>
$$

Our results confirm this. We have found that, by making the ansatz (36), the boundary state equation is indeed solved by such a state. Comparing (62) with our solution leads to the conclusion that

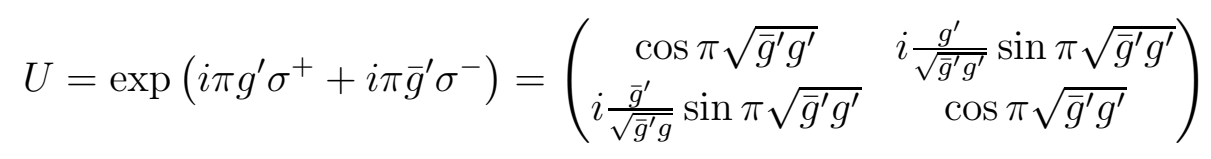

Comparing this with (39) we see that the relationship between coupling constants is

$$
\sin ^{2} \pi \sqrt{\bar{g}^{\prime} g^{\prime}}=\pi^{2} \bar{g} g \quad, \quad \frac{g^{\prime}}{\bar{g}^{\prime}}=\frac{g}{\bar{g}}
$$

As we would expect, since in the special case when either $g=0$ or $\bar{g}=0$, operator ordering does not block the application of the Neuman boundary condition in the exponent, the relation becomes an identity for the other constant $\left(\bar{g}^{\prime}=\bar{g}\right.$ or $g^{\prime}=g$, respectively). (64) does not agree with the transformation of coupling constants found in ref. 35] or 21] and used in [18]. We attribute this to dependence on regularization scheme and regard it as a re-parameterization of coupling constants which should have no effect on the physical meaning of the theory. However, a clear understanding of how correlators are related to the bare coupling constants could be of value in condensed matter applications.

5. Band structure of the spectrum: In ref. 24] it was shown that, in some cases the spectrum of a non-compact boson takes on an interesting band structure. To recall what the band structure is, we begin with translation invariant theories with a free boson. If both boundary conditions are Neumann, for example, the boson has momentum $p$ and energy $E=\frac{p^{2}}{2}$. In our construction, we begin by treating it as a compact boson, whose momenta are quantized, $\frac{1}{\sqrt{2}} p=n$. The presence of the Wilson line term in the action shifts these momenta by a constant, $\frac{1}{\sqrt{2}} p=n+A$ and the energy is $E=(n+A)^{2}$. We see that the spectrum is split into "energy bands". The integer $n$ labels the band and as we vary $A$ over its range (say $-\frac{1}{2}$ to $+\frac{1}{2}$, we sweep through the energies in a given band. Of course, for the free boson with Neumann boundary conditions, combining the bands just recovers the parabolic spectrum $E=\frac{1}{2} p^{2}$ with $p$ varying over the real numbers.

Now, we have observed that the addition of a boundary interaction with discrete translation symmetry changes the boson momenta and energies to $\frac{1}{\sqrt{2}} p=n+\delta(A, n)$ and $E(n, A)=(n+\delta(A, n))^{2}$, respectively. As we sweep $A$ over its range of allowed 
values, $-\frac{1}{2}<A \leq \frac{1}{2}, \frac{1}{\sqrt{2}} p=n+\delta(A, n)$ varies over a range of momenta and $E(n, A)$ varies over the $n$ 'th energy band.

In eqs. (66) and (77) we see that $\delta(A, n)$ does not depend on $n$, reduces to $\delta_{0}=A$ for a boson with Neumann boundary conditions at both boundaries, and otherwise is a function of the coupling constants of the boundary sine-Gordon theory and $A$ given in (7). The example of the system with a Neumann boundary condition plus boundary sine-Gordon with particular values of the coupling constants are shown in fig. (11).

When the translation invariance is broken entirely, as in the case of a Dirichlet boundary condition, we can still think of the position of the D-brane as a parameter analogous to $A$. Translation invariance can be restored by integrating over this parameter. For example, in eq. (154), if the D-brane were placed at $X=x_{0}$, rather than $X=0$, the energy shift would be replaced by

$$
\delta=\frac{1}{2 \pi} \cos ^{-1} \frac{\pi}{2}\left(g e^{i x_{0}}+\bar{g} e^{-i x_{0}}\right) .
$$

This formula is obtained from (7) by putting $\left(g_{1}, \bar{g}_{1}, A_{1}\right) \rightarrow(g, \bar{g}, A)$ and $\left(g_{2}, \bar{g}_{2}, A_{2}\right) \rightarrow$ $\left(-\frac{1}{\pi} e^{i x_{0}},-\frac{1}{\pi} e^{-i x_{0}}, 0\right)$.

As $x_{0}$ varies between $-\pi$ and $\pi, \delta$ sweeps across an "energy band".

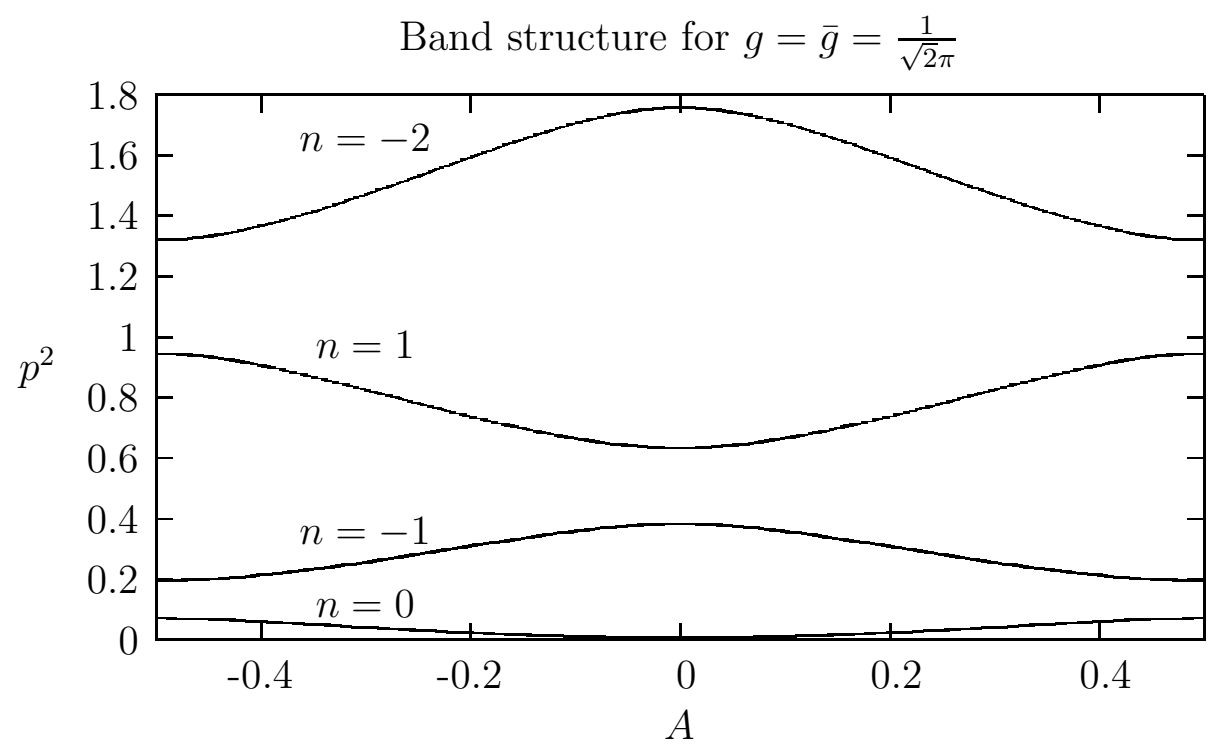

Figure 1: The energy bands in the spectrum of a single boson on a strip with a Neumann boundary condition at one boundary and boundary sine-Gordon interaction on the other. 


\section{Rational radii}

In the boson field theory quantized on the cylinder, the dependence on the compactification radius of the boson resides entirely in the allowed momentum quantum numbers. The total momentum, $\frac{1}{\sqrt{2}}\left(p_{X L}+p_{X R}\right)$ must come in units of integer $/ R$ whereas the dual momentum $\frac{1}{\sqrt{2}}\left(p_{X L}-p_{X R}\right)$ must be integers $R$. So far, we have considered the case of self-dual radius, $R=1$. In this section, we will consider other radii, which are rational numbers $R=\frac{M}{N}$ where $M$ and $N$ are co-prime integers.

It has been shown 29, 20, that conformal boundary states at rational radii can be found by simply choosing that subset of the states contained in the boundary state at the self-dual radius which have the correctly quantized momenta. They further argue that, together with the $\mathrm{SL}(2, \mathrm{C})$ boundary states that we have already discussed, these exhaust the set of possible conformal boundary states of a system with one massless boson in the bulk.

Since, in the fermion description of the theory, the momenta are fermion numbers, this means that these states can be found by projecting the boundary states at the selfdual radius onto sectors with fixed fermion number. If we consider the $X$-boson with the identification

$$
X \sim X+2 \pi \frac{M}{N}
$$

where $M$ and $N$ are mutually prime, then the momentum and wrappings are quantized as

$$
\frac{1}{\sqrt{2}} p_{X}=\frac{1}{\sqrt{2}}\left(p_{X L}+p_{X R}\right)=K \frac{N}{M} \quad, \quad \frac{1}{\sqrt{2}} w_{X}=\frac{1}{\sqrt{2}}\left(p_{X L}-p_{X R}\right)=L \frac{M}{N}
$$

where $K$ and $L$ are integers. From these, we must choose states that are already in the spectrum at the self-dual radius, i.e. where the right-hand-sides of these equations are equal to integers. This means that we can only find states where $K=Q M$ and $L=R N$ with $(Q, R)$ integers. That is,

$$
\frac{1}{\sqrt{2}}\left(p_{X L}+p_{X R}\right)=K \frac{N}{M}=Q N \quad, \quad \frac{1}{\sqrt{2}}\left(p_{X L}-p_{X R}\right)=L \frac{M}{N}=R M
$$

Now, using (25), (28a $),(28 \mathrm{~b})$ and (29), we see that, in terms of the current algebra charges, these imply the constraints

$$
J_{L}^{3}-J_{R}^{3}=Q N \quad, J_{L}^{3}+J_{R}^{3}=R M
$$

where $Q$ and $R$ are any integers. We can impose these as constraints on the boundary state by operating with projection operators,

$$
\left|B_{M / N}, D>=\frac{1}{M N} \sum_{Q=0}^{N-1} \sum_{R=0}^{M-1} e^{2 \pi i \frac{Q}{N}\left(J_{L}^{3}-J_{R}^{3}\right)} e^{2 \pi i \frac{R}{M}\left(J_{L}^{3}+J_{R}^{3}\right)}\right| B D>
$$

Since this projection does not involve the current algebra $J^{a^{\prime}}$ in (30), it does not disturb the Dirichlet boundary condition for the Y-boson. We can also still take the Y-boson to be compact with the self-dual radius. 
The expression in (69) is a sum over the $\mathrm{SL}(2, \mathrm{C})$ boundary states. The state in each term in the sum is of the form

$$
\begin{aligned}
e^{2 \pi i \frac{Q}{N}\left(J_{L}^{3}-J_{R}^{3}\right)} e^{2 \pi i \frac{R}{M}\left(J_{L}^{3}+J_{R}^{3}\right)} \mid B D> & =e^{2 \pi i\left(\frac{Q}{N}+\frac{R}{M}\right) J_{L}^{3}} e^{-i \theta \cdot J_{L}} e^{2 \pi i\left(-\frac{Q}{N}+\frac{R}{M}\right) J_{R}^{3}} \mid N D> \\
& =e^{2 \pi i\left(\frac{Q}{N}+\frac{R}{M}\right) J_{L}^{3}} e^{-i \theta \cdot J_{L}} e^{2 \pi i\left(-\frac{Q}{N}+\frac{R}{M}\right) J_{L}^{3}} \mid N D>
\end{aligned}
$$

This effectively replaces the matrix $U$ by the transformed matrix

$$
U \rightarrow e^{\pi i\left(\frac{Q}{N}-\frac{R}{M}\right) \sigma^{3}} U e^{-\pi i\left(\frac{Q}{N}+\frac{R}{M}\right) \sigma^{3}}=\left(\begin{array}{cc}
e^{-2 \pi i(A+R / M)} \sqrt{1-\pi^{2} g \bar{g}} & -i \pi g e^{2 \pi i Q / N} \\
-i \pi \bar{g} e^{-2 \pi i Q / N} & e^{-2 \pi i(A+R / M)} \sqrt{1-\pi^{2} g \bar{g}}
\end{array}\right)
$$

Thus, each of the boundary states is the boundary state for a boundary sine-Gordon theory with the replacement of parameters

$$
g \rightarrow g e^{2 \pi i Q / N} \quad, \quad A \rightarrow A+R / M
$$

Then, a field theory of a boson on a strip for which $\mid B_{M / N} D>$ in (69) is the correct boundary state has the partition function

$$
Z=\frac{1}{M N} \sum_{Q=0}^{N-1} \sum_{R=0}^{M-1} \int[d X] e^{-S[X, M, N ; Q, R]}
$$

where the Euclidean action is

$$
\begin{aligned}
S=\frac{1}{4 \pi} \int_{0}^{\beta} d \tau \int_{0}^{\pi} d \sigma\left(\partial_{\tau} X(\tau, \sigma)^{2}\right. & \left.+\partial_{\sigma} X(\tau, \sigma)^{2}\right)+i \int_{0}^{\beta} d \tau\left(A+\frac{R}{M}\right) \partial_{\tau} X(\tau, 0) \\
& +\int_{0}^{\beta} d \tau\left(\frac{g}{2} e^{i X(\tau, 0)+2 \pi i \frac{Q}{N}}+\frac{\bar{g}}{2} e^{-i X(\tau, 0)-2 \pi i \frac{Q}{N}}\right)
\end{aligned}
$$

Note that the potential term in the action is invariant under simultaneous translations $X \rightarrow X+2 \pi$-integer $/ \mathrm{N}$ and $Q \rightarrow Q$-integer mod $\mathrm{N}$. Then, we use the prescription of Sect. 1.1 to sum over Bloch wave-number, elongating the period from $X \rightarrow X+2 \pi \frac{1}{N}$ to $X \rightarrow X+2 \pi \frac{M}{N}$. The remaining parameter has the identification $A \sim A+\frac{N}{M}$.

The constraint on the boundary state in (69) is reminiscent of a Gauss' law constraint in a gauge field theory, with the unusual feature that it requires invariance only under discrete subgroups of $U(1)$. It is easy to imagine that such a theory could be an effective field theory for a discrete gauge theory. Such theories are known to arise when a continuous gauge symmetry is not fully spontaneously broken, but leaves a discrete residual gauge group 36 .

\section{Disc Amplitude}

In this section, we will consider the disc correlation functions

$$
<p_{X L}, p_{X R} \mid B>
$$


These are called disc correlation functions because they should reproduce the Euclidean path integral where the two dimensional space-time is a disc, rather than strip, with the boundary interaction living at the boundary of the disc, and the bulk vertex operator $: e^{i p_{X L} X_{L}+i p_{X R} X_{R}}:$ inserted at the center. The result is

$$
2^{\frac{1}{4}}<p_{X L}, p_{X R} \mid B>=\left\{\begin{array}{cc}
{\left[-i U_{12}\right]^{\sqrt{2} p_{X L}}} & p_{X L}=p_{X R}>0 \\
{\left[U_{11}\right]^{\sqrt{2} p_{X L}}} & p_{X L}=-p_{X R}>0 \\
{\left[U_{22}\right]^{-\sqrt{2} p_{X L}}} & -p_{X L}=p_{X R}>0 \\
{\left[-i U_{21}\right]^{-\sqrt{2} p_{X L}}} & p_{X L}=p_{X R}<0 \\
0 & \left|p_{X L}\right| \neq\left|p_{X R}\right|
\end{array}\right.
$$

This formula applies to both cases where either both of $\frac{1}{\sqrt{2}} p_{X L}$ and $\frac{1}{\sqrt{2}} p_{X R}$ are integers or both are half-odd-integers.

We can use eq. (75) to compute the overlap of the boundary state with the state which is a vacuum for all bulk boson oscillators and which is an eigenstate of the average position of the $X$-boson. We define the variables

$$
X_{0}=\frac{1}{\sqrt{2}}\left(x_{L}+x_{R}\right) \quad, \quad \hat{X}_{0}=\frac{1}{\sqrt{2}}\left(x_{L}-x_{R}\right)
$$

and the position eigenstates of these operators as

$$
\left|X_{0}, \hat{X}_{0}>=\frac{1}{2 \pi} \sum_{\pi} e^{-i \frac{1}{\sqrt{2}}\left(p_{X L}+p_{X R}\right) X_{0}-i \frac{1}{\sqrt{2}}\left(p_{X L}-p_{X R}\right) \hat{X}_{0}}\right| p_{X L}, p_{X R}>.
$$

Then, taking the matrix element and doing the geometric sums over momenta obtains

$2^{\frac{1}{4}}<X_{0}, \hat{X}_{0} \mid B>=\frac{1}{2 \pi}\left[\frac{1}{1-U_{11} e^{i \hat{X}_{0}}}+\frac{1}{1-U_{22} e^{-i \hat{X}_{0}}}+\frac{1}{1+i U_{12} e^{i X_{0}}}+\frac{1}{1+i U_{21} e^{-i X_{0}}}-3\right](77)$

This formula can be applied to the case of a non-compact boson, and a compact boson with self-dual and fractional radii. The appropriate expressions are presented in eqs. (10)(12).

In order to illustrate the simplicity of the computation, we spell it out in some detail below. We arrive at this result be computing the state $<p_{X L}, p_{X R}, p_{Y L}=0, p_{Y R}=0 \mid B D>$. The overlap with the zero momentum states for the $Y$-boson with its Dirichlet state gives a factor of $2^{-1 / 4}$. Thus, the $Y$-boson contribution is trivial and we can later cancel it by multiplying by $2^{1 / 4}$.

Recall that $p_{Y L}=\frac{1}{\sqrt{2}}\left(\pi_{1 L}-\pi_{2 L}\right)$ and $p_{Y R}=\frac{1}{\sqrt{2}}\left(\pi_{1 R}-\pi_{2 R}\right)$. For this reason, we need to consider states whose momenta obey $\pi_{1 L}=\pi_{2 L} \equiv \pi_{L}=\frac{1}{\sqrt{2}} p_{X L}$ and $\pi_{1 R}=\pi_{2 R} \equiv \pi_{R}=$ $\frac{1}{\sqrt{2}} p_{X R}$.

Also, recall that, in the NS sector, $\left(\pi_{L}, \pi_{R}\right)$ are both integers, whereas in the $\mathrm{R}$ sector $\left(\pi_{L}, \pi_{R}\right)$ are both half-odd-integers. Both of these possibilities must be considered. Since the boundary states have even fermion number, no further GSO projection is needed. 
The total boson momentum is related to total fermion number,

$$
\begin{aligned}
& \pi_{1 L}=\int \frac{d \sigma}{2 \pi}: \psi_{1 L}^{\dagger} \psi_{1 L}: \quad, \quad \pi_{1 R}=-\int \frac{d \sigma}{2 \pi}: \psi_{1 R}^{\dagger} \psi_{1 R}: \\
& \pi_{2 L}=-\int \frac{d \sigma}{2 \pi}: \psi_{2 L}^{\dagger} \psi_{2 L}: \quad, \quad \pi_{1 R}=\int \frac{d \sigma}{2 \pi}: \psi_{2 R}^{\dagger} \psi_{2 R}:
\end{aligned}
$$

In the NS sector, the fermion vacuum is unique and the fermion number is quantized as integers. The above equations show that the boson momentum is equal to \pm the total fermion number of the states there.

In the $\mathrm{R}$-sector, the vacuum is degenerate and the vacuum states $\mid--->$ have fermion numbers $(-1 / 2,-1 / 2,-1 / 2,-1 / 2)$ and $\pi_{1 L}=-1 / 2, \pi_{1 R}=1 / 2, \pi_{2 L}=1 / 2$ and $\pi_{2 R}=-1 / 2$. Operating fermion operators change these by integers, so that the whole spectrum of fermion numbers and momenta are half-odd-integers.

Then, following ref. [19, we observe that the boson momentum eigenstates which have no boson oscillators excited correspond to fermion states where all fermion states are filled up to a Fermi level. The fermion number of each type of fermions is equal (up to signs) to the momentum of the corresponding boson state.

\subsection{NS Sector}

In the NS-sector, the $\pi_{L}$ and $\pi_{R}$ are integers. We denote the states with these values of boson momentum (and vanishing $Y$-momenta) and no boson oscillators excited by $\mid \pi_{L}, \pi_{R}>$.

We shall require the fermion representation of the boson momentum state in bra-form. The following are the states of the NS sector,

$$
\begin{aligned}
\pi_{L}>0, \pi_{R}>0 \quad<\pi_{L}, \pi_{R} \mid & =<0 \mid \prod_{r=\frac{1}{2}}^{\pi_{L}-\frac{1}{2}}\left(\psi_{2 r}^{\dagger} \psi_{1 r}\right) \prod_{r=\frac{1}{2}}^{\pi_{R}-\frac{1}{2}}\left(\tilde{\psi}_{2 r} \tilde{\psi}_{1 r}^{\dagger}\right) \\
\pi_{L}>0, \pi_{R}<0 \quad<\pi_{L}, \pi_{R} \mid & =<0 \mid \prod_{r=\frac{1}{2}}^{\pi_{L}-\frac{1}{2}}\left(\psi_{2 r}^{\dagger} \psi_{1 r}\right) \prod_{r=\frac{1}{2}}^{-\pi_{R}-\frac{1}{2}}\left(\tilde{\psi}_{1 r} \tilde{\psi}_{2 r}^{\dagger}\right) \\
\pi_{L}<0, \pi_{R}>0 \quad<\pi_{L}, \pi_{R} \mid & =<0 \mid \prod_{r=\frac{1}{2}}^{r-\pi_{L} \frac{1}{2}}\left(\psi_{1 r}^{\dagger} \psi_{2 r}\right) \prod_{r=\frac{1}{2}}^{\pi_{R}-\frac{1}{2}}\left(\tilde{\psi}_{2 r} \tilde{\psi}_{1 r}^{\dagger}\right) \\
\pi_{L}<0, \pi_{R}<0 \quad<\pi_{L}, \pi_{R} \mid & =<0 \mid \prod_{r=\frac{1}{2}}^{-\pi_{L}-\frac{1}{2}}\left(\psi_{1 r}^{\dagger} \psi_{2 r}\right) \prod_{r=\frac{1}{2}}^{-\pi_{R}-\frac{1}{2}}\left(\tilde{\psi}_{1 r} \tilde{\psi}_{2 r}^{\dagger}\right) .
\end{aligned}
$$

The phases of these states are fixed by their overlaps with $\mid D D>$ and $\mid N D>$ which, recalling the known forms of these states in boson variables, must all be either zero or one. 
The boundary state in the NS sector is given in eq. (42) which we copy here for convenience of the reader:

$$
\left|B, D>_{N S}=\frac{1}{\sqrt{2}} \prod_{r=\frac{1}{2}}^{\infty} \exp \left[\psi_{-r}^{\dagger} U^{-1} i \sigma^{1} \tilde{\psi}_{-r}-\tilde{\psi}_{-r}^{\dagger} i \sigma^{1} U \psi_{-r}\right]\right| 0>.
$$

Then, we can take the matrix elements of the momentum states and the boundary state to obtain:

$$
\begin{aligned}
& \pi_{L}>0, \pi_{R}>0 \\
& <\pi_{L}, \pi_{R}\left|B, D>_{N S}=\frac{1}{\sqrt{2}}<0\right| \prod_{r=\frac{1}{2}}^{\pi_{L}-\frac{1}{2}}\left(\psi_{2 r}^{\dagger}+\tilde{\psi}_{a,-r}^{\dagger}\left[i \sigma^{1} U\right]_{a 2}\right)\left(\psi_{1 r}+\left[U^{-1} i \sigma^{1}\right]_{1 a} \tilde{\psi}_{a,-r}\right) . \\
& \prod_{r=\frac{1}{2}}^{\pi_{R}-\frac{1}{2}}\left(\tilde{\psi}_{2 r}-\left[i \sigma^{1} U\right]_{2 a} \psi_{a,-r}\right)\left(\tilde{\psi}_{1 r}^{\dagger}-\psi_{a,-r}^{\dagger}\left[U^{-1} i \sigma^{1}\right]_{a 1}\right) \mid 0> \\
& =\frac{1}{\sqrt{2}}\left(-i U_{12}\right)^{2 \pi_{L}} \delta\left(\pi_{L}-\pi_{R}\right) \\
& \pi_{L}>0, \pi_{R}<0 \\
& <\pi_{L}, \pi_{R}\left|B, D>_{N S}=\frac{1}{\sqrt{2}}<0\right| \prod_{r=\frac{1}{2}}^{\pi_{L}-\frac{1}{2}}\left(\psi_{2 r}^{\dagger}+\tilde{\psi}_{a,-r}^{\dagger}\left[i \sigma^{1} U\right]_{a 2}\right)\left(\psi_{1 r}+\left[U^{-1} i \sigma^{1}\right]_{1 a} \tilde{\psi}_{a,-r}\right) . \\
& \prod_{r=\frac{1}{2}}^{-\pi_{R}-\frac{1}{2}}\left(\tilde{\psi}_{1 r}-\left[i \sigma^{1} U\right]_{1 a} \psi_{a,-r}\right)\left(\tilde{\psi}_{2 r}^{\dagger}-\psi_{a,-r}^{\dagger}\left[U^{-1} i \sigma^{1}\right]_{a 2}\right) \mid 0> \\
& =\frac{1}{\sqrt{2}}\left(U_{11}\right)^{2 \pi_{L}} \delta\left(\pi_{L}+\pi_{R}\right) \\
& \pi_{L}<0, \pi_{R}>0 \\
& \begin{aligned}
<\pi_{L}, \pi_{R} \mid B, D>_{N S}= & \frac{1}{\sqrt{2}}<0 \mid \prod_{r=\frac{1}{2}}^{-\pi_{L}-\frac{1}{2}}\left(\psi_{1 r}^{\dagger}+\tilde{\psi}_{a,-r}^{\dagger}\left[i \sigma^{1} U\right]_{a 1}\right)\left(\psi_{2 r}+\left[U^{-1} i \sigma^{1}\right]_{2 a} \tilde{\psi}_{a,-r}\right) \\
& \prod_{r=\frac{1}{2}}^{\pi_{R}-\frac{1}{2}}\left(\tilde{\psi}_{2 r}-\left[i \sigma^{1} U\right]_{2 a} \psi_{a,-r}\right)\left(\tilde{\psi}_{1 r}^{\dagger}-\psi_{a,-r}^{\dagger}\left[U^{-1} i \sigma^{1}\right]_{a 1}\right) \mid 0> \\
= & \frac{1}{\sqrt{2}}\left(U_{22}\right)^{-2 \pi_{L}} \delta\left(\pi_{L}+\pi_{R}\right)
\end{aligned}
\end{aligned}
$$




$$
\begin{aligned}
\pi_{L}<0, \pi_{R}<0 & \\
<\pi_{L}, \pi_{R} \mid B, D>_{N S}= & \frac{1}{\sqrt{2}}<0 \mid \prod_{r=\frac{1}{2}}^{-\pi_{L}-\frac{1}{2}}\left(\psi_{1 r}^{\dagger}+\tilde{\psi}_{a,-r}^{\dagger}\left[i \sigma^{1} U\right]_{a 1}\right)\left(\psi_{2 r}+\left[U^{-1} i \sigma^{1}\right]_{2 a} \tilde{\psi}_{a,-r}\right) . \\
& \prod_{r=\frac{1}{2}}^{-\pi_{R}-\frac{1}{2}}\left(\tilde{\psi}_{1 r}-\left[i \sigma^{1} U\right]_{1 a} \psi_{a,-r}\right)\left(\tilde{\psi}_{2 r}^{\dagger}-\psi_{a,-r}^{\dagger}\left[U^{-1} i \sigma^{1}\right]_{a 2}\right) \mid 0> \\
= & \frac{1}{\sqrt{2}}\left(-i U_{21}\right)^{-2 \pi_{L}} \delta\left(\pi_{L}-\pi_{R}\right)
\end{aligned}
$$

This summarizes the overlap of momentum eigenstates with the boundary state in the NS sector.

\subsection{R-sector}

In the R-sector, the momenta are half-odd-integral and the momentum states of the $X$ boson are

$$
\begin{aligned}
& \pi_{L}>0, \pi_{R}>0,<\pi_{L}, \pi_{R}|=<+--+| \prod_{n=1}^{\pi_{L}-\frac{1}{2}}\left(\psi_{2 n}^{\dagger} \psi_{1 n}\right) \prod_{n=1}^{\pi_{R}-\frac{1}{2}}\left(\tilde{\psi}_{2 n} \tilde{\psi}_{1 n}^{\dagger}\right) \\
& \pi_{L}>0, \pi_{R}<0,<\pi_{L}, \pi_{R}|=i<++--| \prod_{n=1}^{\pi_{L}-\frac{1}{2}}\left(\psi_{2 n}^{\dagger} \psi_{1 n}\right) \prod_{n=1}^{-\pi_{R}-\frac{1}{2}}\left(\tilde{\psi}_{1 n} \tilde{\psi}_{2 n}^{\dagger}\right) \\
& \pi_{L}<0, \pi_{R}>0,<\pi_{L}, \pi_{R}|=-i<--++| \prod_{n=1}^{-\pi_{L}-\frac{1}{2}}\left(\psi_{1 n}^{\dagger} \psi_{2 n}\right) \prod_{n=1}^{\pi_{R}-\frac{1}{2}}\left(\tilde{\psi}_{2 n} \tilde{\psi}_{1 n}^{\dagger}\right) \\
& \pi_{L}<0, \pi_{R}<0,<\pi_{L}, \pi_{R}|=<-++-| \prod_{n=1}^{-\pi_{L}-\frac{1}{2}}\left(\psi_{1 n}^{\dagger} \psi_{2 n}\right) \prod_{n=1}^{-\pi_{R}-\frac{1}{2}}\left(\tilde{\psi}_{1 n} \tilde{\psi}_{2 n}^{\dagger}\right)
\end{aligned}
$$

Here, again, the phases have been adjusted so that the matrix elements of these states with the $\mid D D>$ and $\mid N D>$ are always either one or zero, just as the phases of the boson states (21a -(21b) (with $A=0$ ) with boson momentum states would be.

The R-sector boundary state is given in eq. (43) which we copy here for the reader's convenience:

$$
\left|B D>_{R}=2^{-\frac{1}{2}} \prod_{n=1}^{\infty} \exp \left[\psi_{-n}^{\dagger} U^{-1} i \sigma^{1} \tilde{\psi}_{-n}-\tilde{\psi}_{-n}^{\dagger} i \sigma^{1} U \psi_{-n}\right] \exp \left[\psi_{0}^{\dagger} U^{-1} i \sigma^{1} \tilde{\psi}_{0}\right]\right|-+-+>\text {. }
$$


Then, the matrix elements are taken as

$$
\begin{aligned}
\pi_{L}>0, \pi_{R}>0 & \\
<\pi_{L}, \pi_{R} \mid B, D>_{R}= & \frac{1}{\sqrt{2}}<+--+\mid \prod_{n=1}^{\pi_{L}-\frac{1}{2}}\left(\psi_{2, n}^{\dagger}+\tilde{\psi}_{a,-n}^{\dagger}\left[i \sigma^{1} U\right]_{a 2}\right)\left(\psi_{1, n}+\left[U^{-1} i \sigma^{1}\right]_{1 a} \tilde{\psi}_{a,-n}\right) \\
& \prod_{n=1}^{\pi_{R}-\frac{1}{2}}\left(\tilde{\psi}_{2, n}-\left[i \sigma^{1} U\right]_{2 a} \psi_{a,-n}\right)\left(\tilde{\psi}_{1, n}^{\dagger}-\psi_{a,-n}^{\dagger}\left[U^{-1} i \sigma^{1}\right]_{a 1}\right) \\
& \quad \exp \left[\psi_{0}^{\dagger} U^{-1} i \sigma^{1} \tilde{\psi}_{0}\right] \mid-+-+> \\
= & \frac{1}{\sqrt{2}}\left(-i U_{12}\right)^{2 \pi_{L}} \delta\left(\pi_{L}-\pi_{R}\right)
\end{aligned}
$$

$$
\begin{aligned}
& \pi_{L}>0, \pi_{R}<0 \\
& <\pi_{L}, \pi_{R}\left|B, D>_{R}=\frac{i}{\sqrt{2}}<++--\right| \prod_{n=1}^{\pi_{L}-\frac{1}{2}}\left(\psi_{2, n}^{\dagger}+\tilde{\psi}_{a,-n}^{\dagger}\left[i \sigma^{1} U\right]_{a 2}\right)\left(\psi_{1, n}+\left[U^{-1} i \sigma^{1}\right]_{1 a} \tilde{\psi}_{a,-n}\right) . \\
& -\pi_{R}-\frac{1}{2} \\
& \prod_{n=1}^{2}\left(\tilde{\psi}_{1, n}-\left[i \sigma^{1} U\right]_{1 a} \psi_{a,-n}\right)\left(\tilde{\psi}_{2, n}^{\dagger}-\psi_{a,-n}^{\dagger}\left[U^{-1} i \sigma^{1}\right]_{a 2}\right) \\
& \exp \left[\psi_{0}^{\dagger} U^{-1} i \sigma^{1} \tilde{\psi}_{0}\right] \mid-+-+> \\
& =\frac{1}{\sqrt{2}}\left(U_{22}\right)^{2 \pi_{L}} \delta\left(\pi_{L}+\pi_{R}\right) \text {, }
\end{aligned}
$$

$$
\begin{aligned}
\pi_{L}<0, \pi_{R}>0 & \\
<\pi_{L}, \pi_{R} \mid B, D>_{R}=- & \frac{i}{\sqrt{2}}<--++\mid \prod_{n=1}^{-\pi_{L}-\frac{1}{2}}\left(\psi_{1, n}^{\dagger}+\tilde{\psi}_{a,-n}^{\dagger}\left[i \sigma^{1} U\right]_{a 1}\right)\left(\psi_{2 r}+\left[U^{-1} i \sigma^{1}\right]_{2 a} \tilde{\psi}_{a,-n}\right) . \\
& \prod_{n=1}^{\pi_{R}-\frac{1}{2}}\left(\tilde{\psi}_{2, n}-\left[i \sigma^{1} U\right]_{2 a} \psi_{a,-n}\right)\left(\tilde{\psi}_{1, n}^{\dagger}-\psi_{a,-n}^{\dagger}\left[U^{-1} i \sigma^{1}\right]_{a 1}\right) \\
= & \frac{1}{\sqrt{2}}\left(U_{11}\right)^{-2 \pi_{L}} \delta\left(\pi_{L}+\pi_{R}\right),
\end{aligned}
$$




$$
\begin{aligned}
\pi_{L}<0, \pi_{R}<0 & \\
<\pi_{L}, \pi_{R} \mid B, D>_{R}= & \frac{1}{\sqrt{2}}<-++-\mid \prod_{n=1}^{-\pi_{L}-\frac{1}{2}}\left(\psi_{1, n}^{\dagger}+\tilde{\psi}_{a,-n}^{\dagger}\left[i \sigma^{1} U\right]_{a 1}\right)\left(\psi_{2 r}+\left[U^{-1} i \sigma^{1}\right]_{2 a} \tilde{\psi}_{a,-n}\right) . \\
& \prod_{n=1}^{-\pi_{R}-\frac{1}{2}}\left(\tilde{\psi}_{1, n}-\left[i \sigma^{1} U\right]_{1 a} \psi_{a,-n}\right)\left(\tilde{\psi}_{2, n}^{\dagger}-\psi_{a,-n}^{\dagger}\left[U^{-1} i \sigma^{1}\right]_{a 2}\right) \\
= & \frac{1}{\sqrt{2}}\left(-i U_{21}\right)^{-2 \pi_{L}} \delta\left(\pi_{L}-\pi_{R}\right) .
\end{aligned}
$$

We see that the overlap of momentum states with $R$ states has a very similar form to the overlaps with NS states.

\section{Conclusions}

The critical theory which we have discussed in this Paper should be difficult to produce in a realistic system. The reason for this is that, when the parameters are tuned so that precisely the exactly marginal boundary operator is allowed by the periodicity of the boson, a bulk operator with the same periodicity is allowed in the bulk. Moreover, $: e^{i X}:$ is a relevant perturbation in the bulk, it drives a Kosterlitz Thouless transition which gaps the spectrum there, nullifying our possibility of finding the critical theory on the boundary.

In string theory, this is the Hagedorn behavior. When a coordinate of the bosonic string is compactified and the radius is lowered from infinity, we expect to see a phase transition when $R=\sqrt{2}$, before we arrive at the self-dual value $R=1$. In the string theory, this is fixed by going to the superstring where super-partners of the boson provide stability. It would be interesting to understand this better in the context of our present analysis.

Further, although the self-dual radius $R=1$ lies in the unstable regime, a large number of the fractional radii $R=M / N$ do not. On general grounds, we expect that their critical states are less stable than the self-dual state - they contain fewer states, so should have smaller entropy and therefore larger free energy - they would avoid the Kosterlitz-Thouless transition in the bulk.

The boundary sine-Gordon theories which have fractional periods are apparently nonlocal. We have presented them as theories at the dual radius with the addition of a discrete gauge invariance. Such theories are indeed known to arise in models of Josephson-junction arrays [37]-39] and whether the conformal field theories with rational radii can be realized there is an interesting question.

The fermionic boundary states are simple and easy to work with. It should be straightforward to use them to check some important previous computations which used other techniques. One example is the closed string emission amplitudes for the rolling tachyon which were computed in [40, and should easily be accessible in the fermion picture. 
Acknowledgement: The authors thank Volker Schomerus and Ian Affleck for extensive and informative discussions. This work is supported in part by NSERC of Canada, by the Pacific Institute for Theoretical Physics and by KOSEF of Korea through CQUeST (Center for Quantum Space Time). The work of TL is also supported by Kangwon National University through the Research Fund for 2003 Faculty Research Abroad.

\section{References}

[1] A. Schmid, Phys.Rev.Lett. 51, 1506 (1983).

[2] F. Guinea, V. Hakim and A. Muramatsu, "Diffusion and localization of a particle in a periodic potential coupled to a dissipative environment," Phys. Rev. Lett. 54, 263 (1985).

[3] M.P.A. Fisher and W. Zwerger, "Quantum Brownian motion in a periodic potential," Phys.Rev. B32, 4190 (1985).

[4] C. G. Callan and L. Thorlacius, "Open String Theory As Dissipative Quantum Mechanics," Nucl. Phys. B 329, 117 (1990).

[5] L.I. Glazman and A.I. Larkin, "New quantum phases in a one-dimensional junction array," Phys.Rev.Lett. 79, 3736 (1997) arXiv:cond-mat/9809118.

[6] R. Fazio and H. van der Zant, "Quantum Phase transitions and Vortex dynamics in Superconducting networks", Phys. Rep. 355, 235 (2001)

[7] D. Giuliano and P. Sodano, "Effective boundary field theory for a Josephson junction chain with a weak link", Nucl. Phys. B711, 480-504, (2005) arXiv:cond-mat/0501378.

[8] C. G. Callan and D. Freed, "Phase diagram of the dissipative Hofstadter model," Nucl. Phys. B 374, 543 (1992) arXiv:hep-th/9110046.

[9] C. G. Callan, A. G. Felce and D. E. Freed, "Critical theories of the dissipative Hofstadter model," Nucl. Phys. B 392, 551 (1993) arXiv:hep-th/9202085.

[10] I. Affleck and A. W. W. Ludwig, "Critical Theory Of Overscreened Kondo Fixed Points," Nucl. Phys. B 360, 641 (1991).

[11] I. Affleck and A. W. W. Ludwig, "The Kondo Effect, Conformal Field Theory And Fusion Rules," Nucl. Phys. B 352, 849 (1991).

[12] C. L. Kane and M. P. A. Fisher, "Transmission through barriers and resonant tunneling in an interacting one-dimensional electron gas," Phys.Rev. B46, 15233 (1992).

[13] C.L. Kane and M.P.A. Fisher, "Contacts and Edge State Equilibration in the Fractional Quantum Hall Effect" cond-mat/9506116 
[14] M. Oshikawa, C. Chamon and I. Affleck, "Junctions of three quantum wires," Phys. Rev. Lett. 91, 206403 (2003) arXiv:cond-mat/0509675.

[15] E. Witten, "On background independent open string field theory," Phys. Rev. D 46, 5467 (1992) arXiv:hep-th/9208027.

[16] S. L. Shatashvili, "Comment on the background independent open string theory," Phys. Lett. B 311, 83 (1993) arXiv:hep-th/9303143.

[17] S. L. Shatashvili, "On the problems with background independence in string theory," Alg. Anal. 6, 215 (1994) arXiv:hep-th/9311177.

[18] A. Sen, "Rolling tachyon," JHEP 0204, 048 (2002) arXiv:hep-th/0203211.

[19] T. Lee and G. W. Semenoff, "Fermion representation of the rolling tachyon boundary conformal field theory," JHEP 0505, 072 (2005) arXiv:hep-th/0502236.

[20] M. R. Gaberdiel and A. Recknagel, "Conformal boundary states for free bosons and fermions," JHEP 0111, 016 (2001) arXiv:hep-th/0108238.

[21] A. Recknagel and V. Schomerus, "Boundary deformation theory and moduli spaces of D-branes," Nucl. Phys. B 545, 233 (1999) arXiv:hep-th/9811237.

[22] C. Ahn and R. I. Nepomechie, "Finite size effects in the XXZ and sine-Gordon models with two boundaries," Nucl. Phys. B 676 (2004) 637 arXiv:hep-th/0309261.

[23] C. Ahn, Z. Bajnok, R. I. Nepomechie, L. Palla and G. Takacs, "NLIE for hole excited states in the sine-Gordon model with two boundaries," Nucl. Phys. B 714, 307 (2005) arXiv:hep-th/0501047.

[24] J. Polchinski and L. Thorlacius, "Free fermion representation of a boundary conformal field theory," Phys. Rev. D 50, 622 (1994) arXiv:hep-th/9404008.

[25] C. G. . Callan, I. R. Klebanov, A. W. W. Ludwig and J. M. Maldacena, "Exact solution of a boundary conformal field theory," Nucl. Phys. B 422, 417 (1994) arXiv:hep-th/9402113.

[26] K. R. Kristjansson and L. Thorlacius, "Correlation functions in a c = 1 boundary conformal field theory," JHEP 0501, 047 (2005) arXiv:hep-th/0412175.

[27] I. R. Klebanov and A. M. Polyakov, "Interaction of discrete states in two-dimensional string theory," Mod. Phys. Lett. A 6, 3273 (1991) arXiv:hep-th/9109032.

[28] N. Seiberg and E. Witten, "Spin Structures In String Theory," Nucl. Phys. B 276, 272 (1986).

[29] M. R. Gaberdiel, A. Recknagel and G. M. T. Watts, "The conformal boundary states for SU(2) at level 1," Nucl. Phys. B 626, 344 (2002) arXiv:hep-th/0108102. 
[30] V. Schomerus, "Non-compact string backgrounds and non-rational CFT," arXiv:hep-th/0509155

[31] M. R. Gaberdiel, "2D conformal field theory and vertex operator algebras," arXiv:hep-th/0509027

[32] F. Larsen, A. Naqvi and S. Terashima, "Rolling tachyons and decaying branes," JHEP 0302, 039 (2003) arXiv:hep-th/0212248.

[33] N. R. Constable and F. Larsen, "The rolling tachyon as a matrix model," JHEP 0306, 017 (2003) arXiv:hep-th/0305177.

[34] M. R. Gaberdiel and M. Gutperle, "Remarks on the rolling tachyon BCFT," JHEP 0502, 051 (2005) arXiv:hep-th/0410098.

[35] H. Kogetsu and S. Teraguchi, "Massless boundary sine-Gordon model coupled to external fields," JHEP 0501, 048 (2005) arXiv:hep-th/0410197.

[36] L. M. Krauss and F. Wilczek, "Discrete gauge symmetry in continuum theories," Phys. Rev. Lett. 62, 1221 (1989).

[37] B. Doucot, M.V. Feigel'man, L.B. Ioffe, "Topological order in the insulating Josephson junction array", Phys. Rev. Lett. 90, 107003 (2003), cond-mat/0211146

[38] L.B. Ioffe, M.V. Feigel'man, "Possible realization of an ideal quantum computer in Josephson junction array", Phys. Rev B66, 224503 (2002), cond-mat/0205186.

[39] B. Doucot, L. B. Ioffe and J. Vidal, "Discrete non-Abelian gauge theories in twodimensional lattices and their realizations in Josephson-junction arrays,", Phys. Rev. B69, 214501 (2004), arXiv:cond-mat/0302104.

[40] N. Lambert, H. Liu and J. Maldacena, "Closed strings from decaying D-branes," arXiv:hep-th/0303139 